\title{
Infinite Time Turing Machines
}

\author{
Joel David Hamkins ${ }^{1}$ and Andy Lewis
}

February 1, 2008

\begin{abstract}
We extend in a natural way the operation of Turing machines to infinite ordinal time, and investigate the resulting supertask theory of computability and decidability on the reals. Every $\Pi_{1}^{1}$ set, for example, is decidable by such machines, and the semi-decidable sets form a portion of the $\Delta_{2}^{1}$ sets. Our oracle concept leads to a notion of relative computability for sets of reals and a rich degree structure, stratified by two natural jump operators.
\end{abstract}

In these days of super-fast computers whose speed seems to be increasing without bound, the more philosophical among us are perhaps pushed to wonder: what could we compute with an infinitely fast computer? By proposing a natural model for supertasks - computations with infinitely many steps - we provide in this paper a theoretical foundation on which to answer this question. Our model is simple: we simply extend the Turing machine concept into transfinite ordinal time. The resulting machines can perform infinitely many steps of computation, and go on to more computation after that. But mechanically they work just like Turing machines. In particular, they have the usual Turing machine hardware; there is still the same smooth infinite paper tape and the same mechanical head moving back and forth according to a finite algorithm, with finitely many states. What is new is the definition of the behavior of the machine at limit ordinal times. The resulting computability theory leads to a notion of computation on the reals, concepts of decidability and semi-decidability for sets of reals as well as individual reals, two kinds of jump-operator, and a notion of relative computability using oracles which gives a rich degree structure on both the collection of reals and the collection of sets of reals. But much remains unknown; we hope to stir interest in these ideas, which have been a joy for us to think about.

\footnotetext{
${ }^{1}$ The research of the first author was partially supported by a grant from the PSCCUNY Research Foundation.
} 
There has been much work in higher recursion theory analyzing wellfounded computations on infinite objects (see e.g. [Sacks]). Much of that theory, however, grows out of the analogy which associates the $\Delta_{1}^{1}$ sets with the finite sets and the $\Pi_{1}^{1}$ sets with the semi-decidable sets. It therefore gives a different analysis than ours, since in our account, the $\Pi_{1}^{1}$ sets will become actually decidable, along with their complements the $\Sigma_{1}^{1}$ sets, and the semi-decidable sets, with the jump operator, will stratify the class of $\Delta_{2}^{1}$ sets.

Various philosophers and physicists have investigated supertasks, or tasks involving infinitely many steps, the first of which is done, for example, in a half second, the next in a quarter second, and so on, so that the entire job is complete in a finite amount of time. Thomson's lamp [Thom, for example, is on between time $t=0$ and $t=1 / 2$, off until $t=3 / 4$, on until $t=7 / 8$, and so on. More useful supertasks, perhaps, have been proposed which determine the truth of an existential number-theoretic question, such as whether there are additional Fermat primes, by ever more rapidly checking the instances of it so that they are all checked in a finite amount of time. What is intriguing about the physicist's analysis of supertasks is that they have been able to construct general relativistic models in which the supertasks can apparently be carried out [Ear \& Nor], Ear], [Pit], [Hog92], Hog94]. The models generally involve an agreement between two observers, one of whom performs the rote steps of computation looking for a counterexample, the other of whom, while flying ever more rapidly around the first observer, waits patiently for what to him will be a finite amount of time for a signal that a counterexample has been found. Earman shows how it can happen that the entire infinite past half-life of one observer is in the causal past of a point in the life of another observer. Hogarth [Hog94] discusses more complicated arrangements in which the truth of any arithmetic statement can be computed. But as we will show in this paper, the supertask concept allows one to compute the truth of even more complicated statements than this. What we are interested in here is not so much finding what is physically possible to compute in a supertask so much as what is mathematically possible. Though the physicists may explain how it is possible to carry out a supertask in a finite amount of time, we, being focused on the algorithm, will nevertheless regard the supertask computations as being infinite in the sense that they involve infinitely many steps of computation.

A word of credit is due. Jeffrey Kidder defined infinite time Turing ma- 
chines in 1989, and he and the first author of this paper worked out the early theory while they were graduate students together at the University of California in Berkeley. The notes sat neglected, unfortunately, in the filing cabinet of the first author, carted from one university to another, but were never forgotten. With a fresh look in 1996, a few of the formerly puzzling questions were answered, and a more advanced theory developed, which we give in this paper.

We will assume complete familiarity with the notions of Turing machines and ordinals and, in describing our algorithms, take the high road to avoid getting bogged down in Turing machine minutiae. We hope the readers will appreciate our saving them from reading what would otherwise resemble computer code.

We begin by describing in section 1 how the new machines work. Next, in section 2, we investigate their power, which lies between $\Pi_{1}^{1}$ and $\Delta_{2}^{1}$, and generalize the classical s-m-n and Recursion theorems to the supertask context. Our analysis of the lengths of supertask computations leads in section 3 to the notions of clockable and writable ordinals. We present in section 4 the supertask halting problems, a lightface and boldface version, and prove the Lost Melody Theorem, which has the intriguing consequence that there are noncomputable functions whose graphs are infinite time decidable. This leads naturally in section 5 to the notion of oracles and the two jump operators corresponding to the two halting problems. We develop in section 6 the basic features of the structure of infinite time degrees; a key feature of this structure, having no classical analog, is the interplay between reals as oracles and sets of reals as oracles. We highlight, in section 7 , the descriptive set-theoretic aspects of our theory and, in section 8, the connections with admissible set theory; it turns out that the supremum of the writable ordinals is a rather large countable ordinal, being recursively inaccessible and indeed indescribable by semi-decidable properties. We conclude the paper by giving five equivalences to the question of whether every clockable ordinal is writable, a question which remains open.

\section{How the Machines Work}

Let us now describe exactly how an infinite time Turing machine works. Like a Turing machine, our supertask machines have a head which moves mechan- 
ically back and forth, reading and writing 0 s and 1 s on a tape according to a finite algorithm $p$. For convenience, we will set up our machines with three separate tapes, one for input, one for scratch work, and one for output.

The machine begins, like a Turing machine, with the head resting in anticipation on the first cell in a special state called the start state. The input is written on the input tape, and the scratch tape and the output tape are filled with zeros. At each step of computation, the head reads the cell values which it overlies, reflects on its state, consults the program about what should be done in such a situation and then carries out the instructions: it writes a 0 or 1 on (any of) the tapes, moves left or right and switches to a new state accordingly. This procedure determines the configuration of the machine at stage $\alpha+1$, given the configuration at stage $\alpha$, for any $\alpha$. It remains to somehow take a limit of these computations in order to identify the configuration of the machine at stage $\omega$ and, more generally, at limit ordinal stages in the supertask computation. To set up such a limit ordinal configuration, the head is plucked from wherever it might have been racing towards, and placed on top of the first cell. And it is placed in a special distinguished limit state. Now we need to take a limit of the cell values on the tape. And we will do this cell by cell according to the following rule: if the values appearing in a cell have converged, that is, if they are either eventually 0 or eventually 1 before the limit stage, then the cell retains the limiting value at the limit stage. Otherwise, in the case that the cell values have alternated from 0 to 
1 and back again unboundedly often, we make the limit cell value 1 . This is equivalent to making the limit cell value the lim sup of the cell values before the limit. This completely describes the configuration of the machine at any limit ordinal stage $\beta$, and the machine can go on computing to $\beta+1, \beta+2$, and so on, eventually taking another limit at $\beta+\omega$ and so on through the ordinals. If at any stage, the machine finds itself in the special halt state, then computation ceases, and whatever is written on the output tape becomes the official output. Otherwise, the infinite time machine will compute endlessly as the ordinals fall one after another through the transfinite hourglass.

In this way every infinite time Turing machine program $p$ determines a function. On input $x$, we can run the machine with program $p$, and, if it halts, there will be some output, which we denote by $\varphi_{p}(x)$. The domain of $\varphi_{p}$ is simply the collection of $x$ which lead to a halting computation. Notice that the natural input for these machines is an infinite binary string $x \in 2^{\omega}$. Thus, the infinite time computable functions are partial functions on Cantor space. In this paper, we will refer to the elements of Cantor space $2^{\omega}$ as 'reals', and think of the computable functions as functions on the reals. In particular, we will denote $2^{\omega}$ by $\mathbb{R}$. By adding extra input tapes, we may have functions of more than one argument, and, in the usual Turing machine argument, these can be simulated by, for example, interleaving the digits of the inputs and using a machine with only one input tape. Also, we will regard 0 and 1 as elements of $\mathbb{R}$, by the convention, for example, in which 0 represents $\langle 0,0,0, \ldots\rangle$ and 1 represents $\langle 1,0,0,0, \ldots\rangle$.

Let us now make a few basic definitions. A partial function $f: \mathbb{R}^{k} \rightarrow \mathbb{R}$ is infinite time computable when there is a program $p$ such that $f=\varphi_{p}$. For simplicity, we will assume by some suitable coding mechanism that a program is represented by a natural number, and furthermore, that every natural number represents a program. A set of reals $A$ is infinite time decidable when the characteristic function of $A$ is infinite time computable (and for the sake of brevity, we will normally use just the term "decidable," unless we fear some misunderstanding). The set $A$ is infinite time semi-decidable when the function which gives the affirmative values, the function with domain $A$ and constant value 1 , is infinite time computable. Thus, a set is semi-decidable exactly when it is the domain of a computable function, since it is a simple matter to modify a program to change the output to the constant 1 . We can also stratify the computable sets according to how long the computations take. Thus, a set is $\alpha$-decidable when the characteristic function of the set is 
computable by a machine which on any input takes fewer than $\alpha$ many steps. Thus, restricting to the case of finite input and time, a function $f: 2^{<\omega} \rightarrow 2^{<\omega}$ is $\omega$-computable exactly when it is computable in the Turing machine sense.

Now that we have made the initial definitions, let us get started by proving that we may restrict our attention always to the countable ordinals.

Theorem 1.1 Every halting infinite time computation is countable.

Proof: Suppose the supertask computation of program $p$ on input $x$ has run for uncountably many steps without halting. We will show that this computation will never halt. Let us say that a snapshot of a computation is a complete description of the configuration: it specifies the program being used, the state and position of the head and the complete contents of each of the tapes. All this information can be coded in some canonical way into a real. Consider the snapshot of the $\omega_{1}$-stage of computation. We will argue that in fact this very same snapshot occurred earlier, at some countable stage, and that the computation is caught in an infinite loop which will repeat forever. First, observe that at stage $\omega_{1}$ the head is on the first cell in the limit state, as it is at any limit ordinal stage. Next, observe that if the value of any cell is 0 at stage $\omega_{1}$, then there must be some countable stage at which the cell had the value 0 and never subsequently changed. If the cell has the value 1 at stage $\omega_{1}$, then there are two possibilities: either at some stage the cell obtained a value of 1 and was not subsequently changed to a 0 , or else the value of the cell alternated unboundedly often before stage $\omega_{1}$. Now we apply a simple cofinality argument. Since there are only countably many cells, by taking a countable supremum we can find a stage $\alpha_{0}$ where the cells which eventually stabilize have already all stabilized. After this stage, the only cells which change are the ones which will change cofinally often, and so there must be a sequence of countable ordinals $\alpha_{0}<\alpha_{1}<\alpha_{2}<\ldots$ such that between $\alpha_{n}$ and $\alpha_{n+1}$ all the cells which change at all after $\alpha_{n}$ have changed value at least once by $\alpha_{n+1}$. Let $\delta=\sup _{n} \alpha_{n}$. At this limit stage, the head is on the first cell in the limit state, the cells which stabilize before $\omega_{1}$ have stabilized before $\delta$, and the cells which change values unboundedly often before $\omega_{1}$ have changed values unboundedly often before $\delta$. Thus, the snapshot at stage $\delta$ is the same as the snapshot at stage $\omega_{1}$. Thus, the computation has repeated itself. And moreover it has done so in a very strong way: none of the cells which are zero at stage $\delta$ will ever again turn to one, 
so the computation will cycle endlessly. Limit stages in which the repeating snapshot has repeated unboundedly often will again be the very same repeating snapshot, and so the computation is caught in an infinite repeating loop. $\square$

Let us point out that it is possible for a computation to repeat-for the very same snapshot to occur twice during a computation-but for the computation nevertheless eventually to escape this infinite loop. This could occur, for example, if the limit of the repeating snapshots is not the same snapshot again. Thus, after repeating $\omega$ many times, the program could escape the loop and go on to compute something else or even halt. Let us say, then, officially, that a computation repeats itself only when the very same snapshot occurs at two limit ordinal stages, and that between these stages the cells which are 0 at the limit never subsequently turn to 1 (we allow the 1 s to turn to 0 and back again). This is equivalent to requiring that the limit of the repeating snapshots is again the very same repeating snapshot. Such computations, therefore, are truly caught in an infinite loop.

Corollary 1.2 Every infinite time computation either halts or repeats itself in countably many steps.

Proof: The previous proof shows that if a computation does not halt, then the snapshot of the machine configuration at stage $\omega_{1}$ appears earlier, and, in fact, appears unboundedly often before $\omega_{1}$, since $\alpha_{0}$ can be chosen arbitrarily large below $\omega_{1}$. Moreover, since these snapshots occurred beyond $\alpha_{0}$, none of the $0 \mathrm{~s}$ in the repeating snapshot ever again turns to 1 . So a non-halting computation repeats itself by some countable stage.

\section{The Power of Infinite Time Machines}

How powerful are these machines? Perhaps the first thing to notice is that the halting problem for Turing machines is infinite time decidable. This is true because with an infinite time Turing machine one can simulate an ordinary Turing machine computation. Either the simulation halts in finitely many steps, or else after $\omega$ many steps the machine reaches the limit state, and so by giving the output Yes or No, respectively, in these two situations, the halting problem is solved. Thus infinite time Turing machines are more powerful 
than ordinary Turing machines: they can decide sets which are undecidable by Turing machines. The next theorem greatly improves on this.

Theorem 2.1 The truth of any arithmetic statement is infinite time decidable.

Proof: It is easy to see by an inductive argument on formulas that first order arithmetic truth is decidable: given a statement $\exists n \varphi(n, \vec{x})$, one simply directs the machine to try out all the possible values of $n$ and test the truth of $\varphi(n, \vec{x})$.

But we can do much better even than this. In the next theorem we will introduce the first argument which really seems to use the full computational power of these machines. A relation $\triangleleft$ on a subset of $\omega$ can be coded by the real $x$ such that $x(\langle n, k\rangle)=1$ exactly when $n \triangleleft k$, where $\langle\cdot, \cdot\rangle$ is some canonical pairing function. In this way every real $x$ codes some relation $\triangleleft$. Let $W O$ be the set of reals coding well-orders. It is well known that WO is a complete $\Pi_{1}^{1}$ set, in the sense that if $A$ is another $\Pi_{1}^{1}$ set then there is a recursive function $f$ on reals such that $x \in A \Longleftrightarrow f(x) \in W O$.

Count-Through Theorem 2.2 WO is infinite time decidable.

Proof: Suppose we are given a real $x$ on the input tape of an infinite time Turing machine. We will describe a supertask algorithm which will determine if this real codes a well order. Certainly in $\omega$ many steps we can determine if the real codes a relation which is reflexive, transitive, and antisymmetric. This involves simply checking that if $x$ says that $n$ is related to $k$ and that $k$ is related to $r$, then it also says that $n$ is related to $r$, and so on. Every instance of this can be systematically checked. Thus, we may assume that the real $x$ survives this first test, and therefore codes a linear order, before continuing further. Next, in $\omega$ many steps, we can find the particular natural number $n$ which is the least element in the relation coded by $x$. This can be done by first, keeping a current guess written on the scratch tape, updating it every time a number is found which precedes it in the relation coded by $x$, and second, flashing a flag on and then off again every time we change the guess. In the limit, if the flag is on, it means that we changed our guess infinitely often, and thus the real $x$ does not code a well-order. If the flag 
is not on, then it must be that the minimal element $n$ of the relation is sitting on the scratch tape, having survived all challengers. In another $\omega$ many steps, we can go through the relation and erase all mention of the number $n$ from the field of the relation. This produces a real coding a relation with a smaller field. Now we simply iterate this. That is, in $\omega$ many steps, first find the least element of the relation coded by the real currently on the tape, and in another $\omega$ many steps, erase all mention of this element, and repeat with the new smaller relation. There is a slight complication at the compound limits (limits of limits) since at such stages we will have a lot of garbage on the scratch tape, but because we were gradually erasing elements from the field of the relation coded by the real on the input tape, the input tape has stabilized to the intersection of those relations, which is exactly what we want there. By flashing a flag on and then off again every time we reach a limit stage, we can recognize a compound limit as a limit stage in which this flag is on, and then in $\omega$ many steps wipe the scratch tape clean before continuing with the algorithm. If the original real codes a relation which is not a well-order, then after its well-founded part has been erased, there will come a stage when it has no least member, and the machine will discover this, since the guesses for the least member at that stage will not converge. If the real does code a well-order, then the elements of the field will gradually be erased until the machine finds that the field of the relation is empty. Thus, in any case the machine will know whether the real codes a well-order, and so $W O$ is decidable.

Corollary 2.3 Every $\Pi_{1}^{1}$ set is infinite time decidable. Hence, every $\Sigma_{1}^{1}$ set is infinite time decidable.

Proof: It is well-known that every $\Pi_{1}^{1}$ set $A$ reduces to $W O$ in the sense that there is some recursive function on reals $f$ such that $x \in A \leftrightarrow f(x) \in W O$. Furthermore, it is clear that an infinite time machine can compute any recursive function on reals. So, fix $A$ and $f$, and consider the algorithm which on input $x$ first computes $f(x)$, and then determines if $f(x) \in W O$. This machine decides whether $x$ is in $A$.

The collection of decidable sets extends further up the analytical hierarchy. A set $A$ is said to be $\beta-\Pi_{1}^{1}$, where $\beta$ is a recursive ordinal coded by 
some recursive relation $E$ on $\omega$, when for each $\alpha \leq \beta$ there is a set $A_{\alpha}$, with $A_{\beta}=\emptyset$, such that, first, $\left\{(k, x) \mid x \in A_{|k|}\right\}$ is $\Pi_{1}^{1}$, where $|k|$ denotes the order-type of $k$ with respect to $E$, and, second, that $x \in A$ iff there is an odd $\alpha<\beta$ such that $x \in \cap_{\delta<\alpha} A_{\delta} \backslash A_{\alpha}$ (see [Dub]). This class of sets extends beyond the $\Pi_{1}^{1}$ sets. We can extend it still further by allowing more complicated relations $E$. Specifically, let us say that a real is writable when there is an infinite time Turing machine which can write it as the final output on input 0 . An ordinal will be regarded as writable when there is a writable real coding that ordinal. We will show later that such ordinals extend far beyond the recursive ordinals. Indeed, their supremum is recursively inaccessible. We can naturally extend the definition of the $\beta-\Pi_{1}^{1}$ sets to the situation when $\beta$ is a writable ordinal.

Suppose now that $A$ is $\beta-\Pi_{1}^{1}$ where $\beta$ is a writable ordinal. Consider the algorithm which first writes the relation $E$ coding $\beta$ on a portion of the scratch tape, and then, using the $\Pi_{1}^{1}$ algorithm makes a list of which $n$ have the property that the input $x$ is in $A_{|n|}$. Finally, by counting through the relation coding $\beta$, the algorithm searches for an odd ordinal $\alpha<\beta$ such that $x \in \cap_{\delta<\alpha} A_{\delta} \backslash A_{\alpha}$. This algorithm will decide whether $x$ is in $A$, giving the following corollary.

Corollary 2.4 If $\beta$ is a writable ordinal, then every $\beta-\Pi_{1}^{1}$ set is decidable.

We aim now to establish a limit on the complexity of decidable sets. In the previous section, we introduced the idea of a snapshot, which is a real which codes the complete description of an infinite time Turing machine while it is computing. Thus, in some canonical manner, a snapshot codes the program the machine is running, the position and state of the head and the complete contents of each of the three tapes. Now let us say that a transfinite sequence of snapshots accords with the program $p$ when each successive snapshot is obtained by running the program $p$ on the configuration described by the previous snapshot and the limit snapshots are obtained from the earlier ones according to the computation rules. We will say that a sequence of snapshots according to a program is settled when the last snapshot has either obtained a halting state or else repeats an earlier snapshot, in the strong sense of a computation repeating itself used after Theorem 1.1. The sequence of snapshots represents in the first case a halting computation and in the second a computation which will endlessly repeat. Thus, a settled sequence of snap- 
shots informs us of the outcome of an infinite time computation. With these ideas we can establish the complexity of such computations.

Complexity Theorem 2.5 The graph of every infinite time computable function is $\Delta_{2}^{1}$. Hence, every decidable set and, indeed, every semi-decidable set, is $\Delta_{2}^{1}$.

Proof: Suppose that $f$ is an infinite time computable function, computed by the program $p$. Now observe that $f(x)=y$ if and only if there is a real $z$ coding a well-ordered sequence of snapshots according to $p$ on input $x$ with output $y$. Thus, the graph of $f$ is $\Sigma_{2}^{1}$. For the other half, remember that by Theorem 1.1 every supertask computation either halts or repeats in countably many steps, and that therefore we only need to consider the settled sequences of snapshots. That is, $f(x)=y$ if and only if every real $z$ coding a well-ordered settled sequence of snapshots according to $p$ on input $x$ shows the output as $y$. Thus the graph of $f$ is also $\Pi_{2}^{1}$, and therefore $\Delta_{2}^{1}$, as desired.

It follows, of course, that every co-semi-decidable set is also $\Delta_{2}^{1}$. Later we will show, using the halting problem for infinite time computations, that there are semi-decidable sets which are not decidable, and therefore also co-semi-decidable sets which are not semi-decidable. Therefore, the semidecidable sets form a proper subclass of the $\Delta_{2}^{1}$ sets, as illustrated in the 
diagram below.

In the later sections we will show how the jump operators actually stratify the class of $\Delta_{2}^{1}$ sets. For now, let us exactly identify the classes of sets which are decidable by algorithms which need to take relatively few limits.

Theorem 2.6 The arithmetic sets are exactly the sets which can be decided by an algorithm using a bounded finite number of limits.

Proof: The inductive argument in Theorem 2.1 essentially shows that a $\Sigma_{n}^{0}$ set can be decided by an algorithm using at most $n$ limits. For the converse direction, let us define the notation $\varphi_{p, \alpha}(x)$ to mean the snapshot of the computation of $\varphi_{p}(x)$ after exactly $\alpha$ many steps of computation. We claim that the relation "s is an initial segment of $\varphi_{p, \omega n}(x)$," that is, $s \subset \varphi_{p, \omega n}(x)$, is arithmetic. Certainly this is true when $n=0$. Now consider $n+1$. Let $p^{\prime}$ be the program which does the same thing when starting that $p$ does at a limit 
stage (thus, for example, $p^{\prime}$ could simply at the first step change to the limit state, and thereafter mimic $p$ ). The only way there can be a 0 on a cell of the tape in the limit $\omega(n+1)$ is when the configuration is set up at stage $\omega n$ so that the cell in question will be 0 from some point on. Thus, $s \subset \varphi_{p, \omega(n+1)}(x)$ if and only if for every $i<\ell(s)$ which corresponds to the value in a cell, $s(i)=0 \longleftrightarrow \exists N \forall m>N \forall t\left[t \subset \varphi_{p, \omega n}(x) \wedge \ell(t)>m \longrightarrow \varphi_{p^{\prime}, m}(t)(i)=0\right]$. The very last clause is equivalent to $\varphi_{p, \omega n+m}(x)(i)=0$, because of the definition of $p^{\prime}$ and the assumption on $t$. Thus, the relation is arithmetic at $\omega(n+1)$, and the claim is proved. The theorem follows, since if $A$ is decided always in $n$ limits by program $p$, then $x \in A$ if and only if $\langle 1\rangle \subset \varphi_{p, \omega n}(x)$, and so $A$ is arithmetic.

Theorem 2.7 The hyperarithmetic sets, the $\Delta_{1}^{1}$ sets, are exactly the sets which can be decided in some bounded recursive ordinal length of time.

Proof: Suppose that $A$ is decidable by program $p$, and the computation $\varphi_{p}(x)$ always halts before the recursive ordinal $\alpha$. We want to prove that $A$ is $\Delta_{1}^{1}$. The ordinal $\alpha$ is coded by some recursive relation $\triangleleft$ on $\omega$, coded by the real $y$. Thus, $x \in A$ if and only if there is a sequence of snapshots of length at most $\alpha$ according to $p$ on input $x$ which shows the computation to halt with output 1 . This is a $\Sigma_{1}^{1}$ property, since whether a real codes a sequence of length at most $\alpha$ is equivalent to whether there is an order isomorphism from the given relation to an initial segment of the relation coded by $y$, and $y$ itself is recursive. Thus, $A$ is $\Sigma_{1}^{1}$. We also know, however, that $x \in A$ if and only if every real which codes a sequence of snapshots according to $p$ on input $x$ which is well-founded at least to $\alpha$, shows the computation to halt with output 1 . This shows that $A$ is $\Pi_{1}^{1}$, and so $A$ is $\Delta_{1}^{1}$, as desired.

Conversely, suppose that $A$ is $\Delta_{1}^{1}$. We want to show that $A$ is infinite time decidable by an algorithm taking some bounded recursive length of time. Every hyperarithmetic set has a recursive Borel code, a recursive well-founded tree where each node is labeled with instructions for building the set from the earlier nodes by taking unions, intersections, or complements, and the minimal nodes are labeled with basic open sets (the whole set $A$ being represented by the top node). Consider the algorithm which, on input $x$, systematically works through this tree and keeps track of whether $x$ is in or out of the set coded by each particular node. Thus, for example, the algorithm determines 
whether $x$ is in each of the open sets at the bottom of the tree, and if a node is labeled with intersection, then the algorithm determines whether $x$ is in the set represented by that particular node by checking whether it has already said that $x$ is in each of the sets represented by the earlier nodes. The top node represents $A$, and so this algorithm will decide whether $x$ is in $A$ or not. Furthermore, since the tree is recursive, the height of the tree is recursive, and so this algorithm will take a bounded recursive length of time to determine the final answer. If the nodes are handled according to their rank in the tree, since it takes $\omega$ many steps to handle each node, the whole algorithm will take $\rho \omega$ many steps, where $\rho$ is the rank of the tree. Since the tree is recursive, this is a recursive ordinal.

Let us conclude this section by proving that two results from classical recursion theory hold also for infinite time Turing machines.

The s-m-n Theorem 2.8 There is a primitive recursive function $s$ defined on the natural numbers such that $\varphi_{p}(\vec{k}, \vec{x})=\varphi_{s(p, \vec{k})}(\vec{x})$.

Proof: The classical proof works in this context just as well. One needs only to check that there is a simple uniform procedure to convert a program $p$ which computes a function of arity $m+n$ into a program $s(p, \vec{k})$ which computes the same function with the first $m$ arguments fixed as $\vec{k}$, thereby computing a function of arity $n$.

The Recursion Theorem 2.9 For any infinite time computable total function $f: \mathbb{N} \rightarrow \mathbb{N}$, there is a program $p$ such that $\varphi_{p}=\varphi_{f(p)}$.

Proof: The classical proof works also for this theorem. Fix the function $f$. Let $r$ be the program which computes the function $\varphi_{r}(q, x)=$ $\varphi_{f(s(q, q))}(x)$. Let $p=s(r, r)$, and observe that $\varphi_{p}(x)=\varphi_{s(r, r)}(x)=\varphi_{r}(r, x)=$ $\varphi_{f(s(r, r))}(x)=\varphi_{f(p)}(x)$, as desired.

So that the reader does not think that all the classical arguments will easily generalize, let us just mention that in the supertask context there are noncomputable functions whose graphs are semi-decidable. Indeed, there is a total constant function which is not computable, but whose graph is nevertheless decidable! This will be proved at the end of section four. 


\section{Clockable Ordinals}

The study of infinite time computations leads inexorably to the desire to know exactly how long the computations take. And this leads to the notion of clockable ordinals. An ordinal $\alpha$ is clockable when there is a program which on input 0 halts in exactly $\alpha$ many steps of computation. That is, the $\alpha^{\text {th }}$ step of computation is the act of changing to the halt state. Any natural number $n$, for example, is clockable - one simply uses a machine which counts through $n$ states before halting. The ordinal $\omega$ is also clockable, since a machine can be programmed, for example, to move the head always to the right, until a limit state is obtained, and then halt. It is not difficult to prove that if $\alpha$ is clockable, then so are $\alpha+1$ and $\alpha+\omega$. These simple ideas show that every

ordinal up to $\omega^{2}$ is clockable, and moreover, if $\alpha$ is clockable, then so is $\alpha+\beta$ for any $\beta<\omega^{2}$. Let us now complete the warm-up for this section by proving that $\omega^{2}$ itself is also clockable. Since $\omega^{2}$ is the first ordinal which is a limit of limit ordinals, we will simply design a machine which can recognize such compound limits. At each limit stage, let the machine flash a flag on and then off again, and then simply wait for the next limit stage. The stage $\omega^{2}$ will be the first time that the machine is in a limit state and sees that the flag is on. (of course, one needs to put this flag, signaling that we are done, on the very first cell of the tape, in order that the machine will not need extra steps after $\left.\omega^{2}\right)$. Direct the machine to halt when this occurs. Thus, $\omega^{2}$ is clockable. The reader may enjoy writing programs to halt at $\omega^{3}$ or $\omega^{\omega^{2}+5}$. The next theorem shows that clockable ordinals are plentiful.

Recursive Clocks Theorem 3.1 Every recursive ordinal is clockable.

Proof: Suppose that $\alpha$ is a recursive ordinal which we would like to show is clockable. By the remarks of the previous paragraph, we may assume that $\alpha$ is a compound limit ordinal, since any ordinal above $\omega^{2}$ is the sum of a compound limit ordinal with an ordinal below $\omega^{2}$. We will describe a supertask algorithm which will halt in exactly $\alpha$ many steps. We will do this by first describing an algorithm which may overshoot $\alpha$, and then making various improvements to ensure that we halt right at $\alpha$.

Since $\alpha$ is recursive, there is a Turing machine which can enumerate a real $x$ coding a relation on $\omega$ with order type $\alpha$. Therefore, there is a machine which can write $x$ on the tape in $\omega$ many steps. By using every other cell of 
the scratch tape, or some such contrivance, we may assume that there is still plenty of room for further computation without overwriting this real $x$. Now we will gradually erase elements from the field of the relation coded by $x$, using the same idea as in the Count-Through Theorem 2.2. In $\omega$ many steps, we can find the particular natural number $n$ which is the least element in the relation coded by $x$, and then, in another $\omega$ many steps, we can erase all mention of this element from the field of the relation. Systematically iterating this, we will successively erase the least element from the relation until there is nothing left in the relation. Then, with one further $\omega$ limit, we will know, while futilely searching for the 'next' least element, that the relation is now empty, and we will halt. This algorithm will take $\omega+(\omega+\omega) \alpha+\omega$ many steps, since we had the first $\omega$ many steps to write the original real, and then for each element of the field of that relation, of which there are $\alpha$ many, we had $\omega$ many steps to find that it was the least element, and another $\omega$ many steps to erase it from the field of the relation. And then we had $\omega$ many steps on top to discover that we were actually done (one needs to put the most important flag, signaling that we are done, on the very first cell of the tape, in order that the machine will not need extra steps after that last limit).

Let us now improve the algorithm and reduce the number of steps required. First, we can combine the initial writing of the relation with the searching for the least element of the relation, and perform these functions simultaneously in the first $\omega$ limit. This produces an algorithm which halts after $(\omega+\omega) \alpha+\omega$ many steps. Second, we can combine the operation of searching for the next least element and erasing mention of the previous least element into one $\omega$ limit, by performing them simultaneously. This produces an algorithm which halts after $\omega \alpha+\omega$ many steps. Now, we will do infinitely many things simultaneously. Rather than searching merely for the least element of the relation, we will search for the least $\omega$ many elements of the relation. This can be done by systematically searching through the relation, and writing all the natural numbers from the field which have been encountered so far on the scratch tape in their order with respect to the relation. Since the relation is a well order, the initial segments of these guesses will eventually stabilize to the least $\omega$ many elements of the relation, and so in the limit we will have written the least $\omega$ many elements of the order, the others having been 'pushed off to infinity', so to say. Thus, in one $\omega$ limit we can simultaneously guess the next $\omega$ least elements of the order, even while we are erasing the previous least $\omega$ many elements from the order. This 
produces an algorithm which will halt after $\alpha+\omega$ many steps. Remember, the $\omega$ on top is the result of not being able to recognize in a limit that we have actually erased everything from the field of the relation. But the following contrivance will allow us to recognize in a limit that the field of the relation has already been erased. At any point in our algorithm, the real coding the relation has some smallest pair $\langle n, k\rangle$ (in the natural ordering of $\mathbb{N}$ ) which has not been erased. Every time we erase what is at that time the smallest pair, let the machine flash a flag on and then off again on the very first cell. In a limit, if there is still something in the field, then there will be a smallest thing in the field which survived through the limit, and so this flag will be 0 . If in a limit the field of the relation is empty, then infinitely often the smallest element was erased, and so this flag will be 1 . Thus, we need not go searching for any elements of the field, since by checking this flag the algorithm can know if there are any elements left in the field. With this additional procedure, the algorithm will halt in exactly $\alpha$ many steps. The ordinal $\alpha$, therefore, is clockable.

Thus the clockable ordinals extend at least up to $\omega_{1}^{C K}$, the supremum of the recursive ordinals. The next theorem shows, however, that they extend even further than this.

Theorem 3.2 The ordinal $\omega_{1}^{C K}+\omega$ is clockable.

Proof: By [Fef \& Spd there is an r.e. relation coding a linear order whose well-founded part is exactly $\omega_{1}^{C K}$. Consider the algorithm which in the first $\omega$ many steps writes this relation on the scratch tape, and then begins counting through it using the algorithm of the previous theorem. At stage $\omega_{1}^{C K}$, the well-founded part of the relation has been completely erased. Thus, in another $\omega$ many steps, the machine will discover that there is no next least element. The algorithm therefore can halt at stage $\omega_{1}^{C K}+\omega$.

We invite the reader to ponder the curious question whether the ordinal $\omega_{1}^{C K}$ itself is clockable; this we will answer later. For now, however, we hope to tease the reader by refusing to state whether the Gap Existence Theorem, following the Speed-up Lemma, hints at the answer to this question or distracts one from it. 
Speed-up Lemma 3.3 If $\alpha+n$ is clockable for some natural number $n$, then $\alpha$ is clockable.

Proof: Certainly the converse holds; by adding extra states one can always make a computation take a certain finite number of steps longer. The hard part is to make a computation shorter. So, suppose that $\alpha+n$ is clockable by the program $p$. By the previous remarks we may assume that $\alpha$ is a limit ordinal. Consider the operation of $p$ on input 0 . At stage $\alpha$, the first $n$ cells of the tapes are set up in such a way, say $\vec{a}$, that the machine will halt in exactly $n$ additional steps. What we will do is design a program which will be able to foresee that the first $n$ cells are like that. And this is how. We will simulate the operation of $p$ on input 0 on every other cell of the scratch tape in such a way that $\omega$ many steps of actual computation will simulate $\omega$ many steps of computation. In the remaining space, we will flash a master flag, on the first cell, every time one of the first $n$ simulated cells is not 0 when the corresponding cell in $\vec{a}$ is 0 . Thus, in a limit, the master flag is 0 only when the first simulated cells have 0s where $\vec{a}$ has 0 s. Secondly, every time each of the first $n$ simulated cells has gotten a 1, when the corresponding cell in $\vec{a}$ has a 1 , we flash a secondary flag (i.e. we flash the flag after all of the cells have done it since the last flash). Thus, in a limit, the secondary flag is 1 when all of the first cells have a 1 which should have a 1 according to $\vec{a}$. Thus, in a limit, the first $n$ cells are set up to make the simulation halt when the master flag is off, and the secondary flag is on. And this can be checked right at the limit, since the head is actually looking at three cells at once - one on each of the tapes. So $\alpha$ is clockable.

Any child who can count to 89 can also count to 63 ; counting to a smaller number is generally considered to be easier. Could this fail for infinite time Turing machines? Could there be gaps in the clockable ordinals - ordinals to which infinite time machines cannot count, though they can count higher? The answer, surprisingly, is Yes.

Gap Existence Theorem 3.4 There are gaps in the clockable ordinals. In fact, the first gap above any clockable ordinal has size $\omega$.

Proof: Suppose $\alpha$ is clockable and $\beta$ is the least non-clockable ordinal above $\alpha$. The ordinal $\beta$ must be a limit ordinal, and, by the Speed-up Lemma 3.3, 
there are no clockable ordinals between $\beta$ and $\beta+\omega$. Let us now show that $\beta+\omega$ is clockable. This will imply that the first gap beyond $\alpha$ has size $\omega$. In order to halt at $\beta+\omega$, our basic strategy will be to recognize $\beta$ as the least stage beyond $\alpha$ at which no infinite time algorithm halts. This recognition will take an extra $\omega$ many steps, and so $\beta+\omega$ will be clockable. Consider the algorithm which simulates the computations of $\varphi_{p}(0)$ for every program $p$. That is, by the contrivance of thinking of the scratch tape and output tape as divided into $\omega$ many scratch tapes and output tapes, we will simulate, for every program $p$, the computation of $p$ on input 0 . By setting things up properly, we can arrange that for every $\omega$ steps of our computation, $\omega$ many steps are performed in each of the simulated computations. Since $\alpha$ is clockable, one of these simulations, for some fixed program $p_{0}$, takes $\alpha$ many steps. After waiting for this program to halt in the simulation, our algorithm will keep careful track of when the simulated programs halt. When a stage is found for which none of the simulations halt, then we have found $\beta$ and we halt. Of course, it took us an extra $\omega$ many steps to recognize that none of the simulated computations halted at that stage, so our computation takes $\beta+\omega$ many steps. Thus, $\beta+\omega$ is clockable.

These gaps are a bit mysterious. The following lemmas reveal a little of their structure.

Big Gaps Theorem 3.5 The gaps in the clockable ordinals become large. Indeed, for every clockable ordinal $\alpha$, there are gaps of size at least $\alpha$ in the clockable ordinals.

Proof: Assume without loss of generality that $\alpha$ is a limit ordinal. What we need to prove is that there are limit ordinals $\beta$ such that no ordinal between $\beta$ and $\beta+\alpha$ is clockable, though there are ordinals beyond this that are clockable. And this is how we will do it. Consider the algorithm which searches for a gap of size $\alpha$. It does this by simulating every program $p$ on input 0 and keeping track of which programs have halted. Whenever it finds a stage at which none of the programs have halted, it starts clocking $\alpha$ on the side. For each step of the simulation, it runs one step of the $\alpha$-clock, and pays attention to determine whether the clock or the gap runs out first. If the gap runs out first, then the clock is reset, and the machine searches for the next gap. If the $\alpha$-clock runs out first, revealing a gap of size $\alpha$, then the machine 
halts. If there are no gaps of size $\alpha$, then this machine will run through all the clockable ordinals, until it finds the first $\alpha$ many non-clockable ordinals above all of the clockable ordinals, and then halts. This is a contradiction since it produces a clockable ordinal above all the clockable ordinals. Hence, gaps of size $\alpha$ must exist lower down.

Many Gaps Theorem 3.6 There are many gaps in the clockable ordinals. Indeed, if $\alpha$ is a writable ordinal, then there are at least $\alpha$ many gaps of size at least $\alpha$ in the clockable ordinals. Moreover, if $\alpha$ is either clockable or writable, then the exact ordinal number of gaps of size at least $\alpha$ is neither clockable nor writable.

Proof: We may again assume that $\alpha$ is a limit ordinal. Recall that a writable ordinal is one which is the order type of a relation coded by a writable real. The key idea of this argument is that clockable and writable ordinals both provide a sort of clock by which to measure the length of time elapsed during a simulated computation. That is, we can keep track of how long a simulation takes either by counting through a relation coding $\alpha$, in the case that $\alpha$ is writable, or by performing one additional step of computation in an $\alpha$-clock, in the case that $\alpha$ is clockable. So, suppose now that $\alpha$ is either clockable or writable, and that the exact number of gaps of size at least $\alpha$ is $\beta$, where $\beta$ is either clockable or writable. Consider the algorithm which simulates the computation of all programs $p$ on input 0, searching for gaps. Each time it finds a gap, it counts, using the $\alpha$-clock, to see if the gap has size at least $\alpha$. If so, then the algorithm counts once on the $\beta$-clock. When the $\beta$-clock runs out, then all the gaps have been counted through (and consequently the algorithm has computed beyond every clockable ordinal). By halting when this occurs, the algorithm halts beyond all the clockable ordinals, a contradiction. Thus, $\beta$ must be neither clockable nor writable.

No Gaps Theorem 3.7 There are no gaps in the writable ordinals.

Proof: Suppose $\alpha$ is a writable ordinal. Thus, there is a program $p$ which on input 0 writes a real $x$ coding a relation $\prec_{x}$ with order-type $\alpha$. If $\beta<\alpha$, then there is some natural number $n$ which is the $\beta^{\text {th }}$ element of the order 
$\prec_{x}$. We can now direct a machine to first write $x$ on the tape, and then delete from the field of the relation every element which is not below $n$ with respect to $\prec_{x}$. After this, the machine has written $\prec_{x} \uparrow n$, which has order type $\beta$, and so $\beta$ is writable.

Before proving the next theorem, let us define a real $x$ to be accidentally writable when it appears on one of the tapes during a computation, but not necessarily as the output of a computation. Similarly, a real $x$ is eventually writable when there is a nonhalting infinite time computation, on input 0 , which eventually writes $\mathrm{x}$ on the output tape; that is, beyond some stage, the real appearing on the output tape is $\mathrm{x}$. Thus, it is clear that every writable real is eventually writable, and every eventually writable real is accidentally writable.

Order-type Theorem 3.8 The classes of clockable and writable ordinals have the same order-type. And this order-type is equal to the supremum of the writable ordinals, which is neither clockable nor writable, though it is eventually writable.

Proof: First we will show that if $\beta$ is clockable, then the order-type of the clockable ordinals up to $\beta$ is writable. We will design a supertask algorithm which will write the relation in which $p$ is less than $q$ when $p$ halts before $q$ on input 0 and both halt in less than $\beta$ many steps. Actually, this relation is a pre-wellorder - two programs are equivalent when they halt at the same stage - and so we actually want to include into the field of the relation only the least element of each equivalence class. This produces a relation $\triangleleft$ whose order-type is the same as the order-type of the clockable ordinals below $\beta$. To write the relation $\triangleleft$, we simply simulate the operation of all programs on input 0 , while simultaneously running a clock for $\beta$. In each block of $\omega$ many steps of computation we simulate one step of computation for each of the programs. At the beginning and also after each limit stage, we also compute one step of the $\beta$-clock. By keeping careful track of which programs have halted, we can gradually write the relation $\triangleleft$ on the output tape. Thus, if program $p$ halts, and then $q$ halts, but the $\beta$-clock is still running, then we will ensure that $p \triangleleft q$ on the output tape (while making sure to include only the least element of each equivalence class in the field). When the $\beta$ clock halts, then we halt, and we have written $\triangleleft$, as desired. It follows from this 
that the order-type of the writable ordinals is at least that of the clockable ordinals.

Now we must show the converse. For the remainder of the paper, let $\lambda$ be the supremum of the writable ordinals. Since there are no gaps in the writable ordinals, $\lambda$ is also the least non-writable ordinal. We must show that there are at least $\lambda$ many clockable ordinals. For this, it suffices to show that whenever $\alpha$ is writable, then there are at least $\alpha$ many clockable ordinals. Consider the algorithm which first writes a real coding $\alpha$ on the tape, and then begins counting through $\alpha$, gradually erasing the field of the relation in order. For each natural number $n$, we could design a program which halts when $n$ is the least element of the field of the relation. That is, for each $n$, we could arrange for a machine $p_{n}$ to halt when it has erased the relation up to $n$. With different values of $n$, these programs will halt at different times, and the order-type of their various halting times will be $\alpha$. So there are at least $\alpha$ many clockable ordinals, and the first part of the theorem is proved.

For the second part, we have already mentioned that $\lambda$ is not writable. Suppose it were clockable. Then we could simulate all the programs $p$ on input 0, keeping track of which computations have halted. Every time we find a stage at which one of the computations halts, we run one step of the computation which clocks $\lambda$. When the clock runs out, we halt. Since there are exactly $\lambda$ many clockable ordinals, this algorithm will halt beyond all the clockable ordinals, a contradiction.

It remains only to show that $\lambda$ is eventually writable. But this is easy. As in the argument above, define that $p \triangleleft q$ when $\varphi_{p}(0)$ halts before $\varphi_{q}(0)$, where again we include only the least program from each equivalence class of all programs halting at the same time. This relation is eventually writable by the algorithm which simply simulates all computations $\varphi_{p}(0)$, and outputs Yes whenever it determines that one program halts before another. Since this relation has order-type $\lambda$, we have proved that $\lambda$ is eventually writable.

Let us now prove a few closure theorems for the clockable ordinals.

Theorem 3.9 If $\alpha$ and $\beta$ are clockable, so are $\alpha+\beta$ and $\alpha \cdot \beta$.

Proof: For addition, it suffices to consider only the case when $\beta$ is at least $\omega^{2}$. This will allow us to be a bit sloppy. Consider the supertask in which 
we first clock $\alpha$, and then erase the tape completely, and then clock $\beta$. This takes $\alpha+\omega+\beta$ many steps, but by our assumption on $\beta$ it follows that $\omega+\beta=\beta$, and consequently our algorithm took $\alpha+\beta$ many steps.

The basic idea for ordinal multiplication is that we can clock through $\beta$, and for each tick of that clock, we clock $\alpha$. This will take $\alpha \cdot \beta$ many steps.

We can also handle large sums of clockable ordinals, of the form $\Sigma \alpha_{p}$, where the sum is taken over a computable set of programs $p$, each of which clocks the corresponding ordinal $\alpha_{p}$. That is, if there is some writable real $x$ such that the sum only includes the programs $p$ such that $x(p)=1$, we refer to the sum $\Sigma \alpha_{p}$ as a computable sum.

Theorem 3.10 The supremum of the clockable ordinals is closed under infinite time computable addition. In this sense, it is supertask inaccessible.

Proof: For the remainder of the paper, let $\gamma$ be the supremum of the clockable ordinals. Suppose $x$ is a writable real coding a sequence of programs $p$ which clock some ordinals $\alpha_{p}$. The intended sum is $\Sigma_{x(p)=1} \alpha_{p}$. Consider the supertask algorithm which first writes $x$ and then runs in turn each program $p$ such that $x(p)=1$, and then halts. This algorithm takes at least $\Sigma_{x(p)=1} \alpha_{p}$ many steps, so $\gamma$ is closed under computable addition.

The next theorem shows that there are long stretches of clockable ordinals without any gaps.

Gapless Blocks Theorem 3.11 There are large gapless blocks of clockable ordinals. Indeed, if $\alpha$ is writable in $\delta$ many steps, then $\delta+\beta$ is clockable for any $\beta \leq \alpha$.

Proof: Suppose $\alpha$ is writable, so that there is a program which writes a real $x$ coding a relation with order-type $\alpha$, in $\delta$ many steps. Now suppose $\beta \leq \alpha$. It suffices to treat the case when $\beta$ is a limit ordinal. There must be some natural number $n$ which is the $\beta^{\text {th }}$ element in the relation coded by $x$. Consider the algorithm which first writes $x$, and then counts through the relation coded by $x$, ignoring (and erasing) any part of the field of the relation at $n$ or above. This will take an additional $\beta$ many steps. By the technique of the Recursive Clocks Theorem 3.1, the algorithm can recognize 
in a limit that the relation has already been completely erased. Thus, $\delta+\beta$ is clockable.

Theorem 3.12 If $\alpha$ is either clockable or writable, then the set of reals coding well orders of length less than $\alpha$ is decidable.

Proof: Given an input $x$, we count through it, using the algorithm of Theorem 2.2, while at the same time counting through $\alpha$, either with a clock, or else by first writing a real coding $\alpha$ and gradually erasing elements from the field, depending on whether $\alpha$ is clockable or writable. By paying attention to which computation runs out first, we can know if the given real codes a well order with length less than $\alpha$.

The following basic question remains open. At the end of the paper we will identify necessary and sufficient conditions for an affirmative answer.

Question 3.13 Is every clockable ordinal writable?

\section{The Infinite Time Halting Problems}

The halting problem, ubiquitous in classical computability theory, has an infinite time analog which we will analyze in this section. Officially, we define the halting problems as the sets $H=\{(p, x) \mid p$ halts on input $x\}$, and, the light-face version, $h=\{p \mid p$ halts on input 0$\}$. We will see later that, unlike their classical analogs, these two sets are not equivalent.

Halting Problem Theorem 4.1 The halting problems $h$ and $H$ are semidecidable but not decidable.

Proof: Clearly the halting problems are semi-decidable: to determine if $\varphi_{p}(x)$ halts, one simply simulates the computation, and if it ever does halt, output Yes; otherwise keep simulating. So both $h$ and $H$ are semi-decidable.

Let us now prove that they are not decidable. Suppose the halting problem $H$ was decided by the infinite time computable function $r$. Thus, 
$r(p, x)=1$ when $\varphi_{p}(x) \downarrow$, and otherwise $r(p, x)=0$. Let $q$ be the program which computes the following function:

$$
\varphi_{q}(p)= \begin{cases}\uparrow & \mathrm{r}(\mathrm{p}, \mathrm{p})=1 \\ 1 & \mathrm{r}(\mathrm{p}, \mathrm{p})=0\end{cases}
$$

Now simply observe that $q$ halts on input $q$ iff $q$ does not halt on input $q$, a contradiction. So $H$ is not decidable.

To see that $h$ is not decidable, we will use the Recursion Theorem. Suppose $h$ was decided by some computable function $r$. By the Recursion Theorem 2.9, there is a program $q$ such that

$$
\varphi_{q}(n)= \begin{cases}\uparrow & \mathrm{r}(\mathrm{q})=1 \\ 1 & \mathrm{r}(\mathrm{q})=0\end{cases}
$$

Thus, taking $n=0$, we see that the program $q$ halts on input 0 exactly when the program $q$ does not halt on input 0 , a contradiction.

We will sometimes want to refer to approximations to the halting problem, and so we define $H_{\alpha}=\{(p, x) \mid p$ halts on input $x$ in fewer than $\alpha$ steps $\}$, and $h_{\alpha}=\{p \mid p$ halts on input 0 in fewer than $\alpha$ steps $\}$. It is clear from the definition that if $\alpha<\beta$ then $H_{\alpha} \subseteq H_{\beta}$. What is more, as we will now prove, this inclusion is strict.

Possible Lengths Theorem 4.2 Infinite time computations come in all possible lengths. Indeed, there is a single program which, on various input, performs a halting computation taking any specified non-zero countable ordinal length of time.

Proof: Consider the following supertask algorithm. On input $x$, if the first digit of $x$ is 1 , then the algorithm searches for the next 1 and halts upon finding it. That will take care of computations of non-zero finite length. Now, if the first digit of $x$ is 0 , and the second digit is 1 , then the remaining input is interpreted as a limit ordinal to be counted through according to the algorithm of Theorem 3.1. That will take care of the computations of limit ordinal length. Finally, if the first two digits of $x$ are both 0 , then the next 1 is searched for and then moved two spaces to the left, while what comes after is interpreted as coding a relation to be counted through according to the algorithm of Theorem 3.1 (leaving that first 1 in place). After this, the 
algorithm counts through the empty space until it finds again that first 1 and then halts. For any limit ordinal $\beta$ and any natural number $n$, this kind of input can be arranged to take exactly $\beta+n$ many steps. Thus, on various input, this program computes for any desired non-zero length of time.

Corollary 4.3 If $\alpha<\beta$ then $H_{\alpha}$ is a proper subset of $H_{\beta}$.

Theorem 4.4 For any limit ordinal $\alpha$, neither $H_{\alpha}$ nor $h_{\alpha}$ is $\alpha$-decidable. But if $\alpha$ is clockable, then both $H_{\alpha}$ and $h_{\alpha}$ are $\alpha$-semi-decidable and $(\alpha+1)$ decidable.

Proof: For the first part of this theorem, simply observe that if the function $r$ in the Halting Problem Theorem 4.1 is computable in fewer than $\alpha$ many steps, so is the function computed by the program $q$ which we defined there. So the contradiction of that argument goes through.

For the second part, assume that $\alpha$ is a clockable limit ordinal, and consider the algorithm which, on input $(p, x)$ runs $p$ on input $x$, while at the same time running a program which clocks $\alpha$. If $p$ halts on $x$ before $\alpha$ is clocked, our machine halts with output 1. This occurs before $\alpha$. Otherwise, our machine never halts. Thus, $H_{\alpha}$ is $\alpha$-semi-decidable. It follows that $h_{\alpha}$ is also $\alpha$-semi-decidable.

Lastly, consider the algorithm which operates as in the above paragraph, except that if the $\alpha$-clock halts, this machine halts with an output of 0 . The program can be arranged so as to recognize that the $\alpha$-clock has halted in the $\alpha^{\text {th }}$ step, thus deciding $H_{\alpha}$, and also $h_{\alpha}$, in fewer than $\alpha+1$ many steps.

Theorem 4.5 If $\alpha$ is writable or clockable, then $H_{\alpha}$ and $h_{\alpha}$ are decidable.

Proof: The previous argument handles the case when $\alpha$ is clockable. So assume now that $\alpha$ is writable, and consider the machine which, upon input $(p, x)$, writes a code for $\alpha$ on the output tape, and then simulates $p$ on input $x$, erasing an element from the coding of $\alpha$ after each step in the program. If $p$ halts on $x$ while there is still a non-empty well-order coded, our machine halts with a 1 . Otherwise, if the well-order is completely erased, our machine halts with a 0 . This clearly allows the machine to decide membership in $H_{\alpha}$. It follows that $h_{\alpha}$ is also decidable. 
Theorem 4.6 The set $h_{\alpha}$ is decidable for every $\alpha$ below the supremum of the clockable ordinals.

Proof: Suppose $\alpha<\beta$, where $\beta$ is the least clockable ordinal above $\alpha$. Since no computations on input 0 can halt between $\alpha$ and $\beta$, it follows that $h_{\alpha}=h_{\beta}$. And we know $h_{\beta}$ is decidable by the previous theorem.

Theorem 4.7 Let $\gamma$ is the supremum of the clockable ordinals; then $H_{\gamma}$ is semi-decidable but not decidable.

Proof: Clearly $H_{\gamma}$ is semi-decidable, since on input $(p, x)$, one simulates the program $p$ on input $x$, while searching for a clockable ordinal which is larger. That is, while simulating $\varphi_{p}(x)$, the program also simulates $\varphi_{q}(0)$ for all programs $q$, and pays attention to when the simulations halt. If one of the computations $\varphi_{q}(0)$ halts after the computation $\varphi_{p}(x)$, then, and only then, may it be concluded that $(p, x)$ is in $H_{\gamma}$. So $H_{\gamma}$ is semi-decidable. It cannot be decidable, because $h=h_{\gamma}$ appears as its $0^{\text {th }}$ slice.

We foreshadowed the next few theorems at the end of the second section. If $A$ is a subset of the plane $\mathbb{R} \times \mathbb{R}$, then the slices of $A$ are the sets $A_{y}=$ $\{z \mid(y, z) \in A\}$. Recall that a settled snapshot sequence for $\varphi_{p}(x)$ is a real that codes a well-ordered sequence of snapshots of the computation of $\varphi_{p}(x)$ whose last snapshot is either a halting snapshot or else is the first repeating snapshot (in the strong sense explained just after Theorem 1.1).

No Uniformization Theorem 4.8 There is a decidable subset of the plane $\mathbb{R} \times \mathbb{R}$, all of whose slices are nonempty, which does not contain the graph of any computable total function.

Proof: The following set will do:

$$
A=\left\{(\langle p, x\rangle, z) \mid z \text { codes a settled snapshot sequence for } \varphi_{p}(x)\right\} .
$$

With a suitable pairing function, we may assume every real has the form $\langle p, x\rangle$, and, since every computation $\varphi_{p}(x)$ has a settled snapshot sequence, every slice of $A$ is non-empty. The set $A$ is certainly decidable, since to verify that a proposed real does really code a settled computation sequence 
is very easy; the machine must merely check that the successor steps of the computation are modeled correctly, and that the limit steps are computed correctly from the earlier snapshots, and that the last snapshot is either in a halting state or is the first time that the snapshots repeat. But there can be no computable function $f$ such that $f(\langle p, x\rangle)$ always gives a settled snapshot sequence for $\varphi_{p}(x)$, since such a function could be easily used to decide $H$ : one would simply compute $f(\langle p, x\rangle)$ and observe if the last snapshot was in a halt state.

We should mention that if a decidable subset $A$ of the plane has an accidentally writable real in each section, or indeed, merely has for every real $y$ an accidentally $y$-writable real in the section $A_{y}$, then $A$ will contain the graph of a computable function. On input $y$, simply search for an accidentally $y$-writable real $z$ such that $(y, z) \in A$. Such a real will eventually be found, and so this algorithm gives a computable total function uniformizing $A$. This shows that some computations $\varphi_{p}(x)$ cannot have even accidentally writable settled snapshot sequences.

Like the previous theorem, the next identifies a surprising divergence from the classical theory. The real $c$ in the theorem is like a forgotten melody that you cannot produce on your own but which you can recognize when someone sings it to you.

Lost Melody Theorem 4.9 There is a real, $c$, such that $\{c\}$ is decidable, but $c$ is not writable. Consequently, there is a constant, total function which is not computable, but whose graph is nevertheless decidable: $f(x)=c$.

Proof: The repeat-point of a computation is the ordinal stage by which it either halts or repeats. Let $\delta$ be the supremum of the repeat-points of the computations of the form $\varphi_{p}(0)$. Thus, $\delta$ is a countable ordinal in $L$. Consequently, by a simple bootstrap argument, there is some smallest $\beta \geq \delta$ such that $L_{\beta+1} \models \beta$ is countable (one can take $\beta=\sup \beta_{n}$ where $\beta_{0}=\delta$ and $\beta_{n}$ is countable first in $L_{\beta_{n+1}}$ ). Thus, since $L_{\beta+1}$ has a canonical well-ordering, there is some real $c \in L_{\beta+1}$ which is least with respect to the canonical $L$ order, such that $c$ codes $\beta$. This is our real $c$. First, we will argue that $c$ is not writable. Indeed, it is not even accidentally writable. If it were, then we could solve the halting problem $h$ by searching for an accidentally writable real that codes an ordinal large enough to see the repeat-point of the computation in question. Since $c$ codes $\beta$, which is as large as $\delta$, the real $c$ is 
large enough, and so our algorithm will succeed. This contradicts the fact that $h$ is not decidable. Second, we will argue that $\{c\}$ is decidable. Given a real $z$, we must decide if $z=c$ or not. First, we can determine whether $z$ codes an ordinal or not. Suppose that $z$ codes $\alpha$. Next, we can determine whether this ordinal is larger than $\delta$ by simulating every computation $\varphi_{p}(0)$ along the order given by $z$, and determining if we reach the repeat-point before running out of room. Now comes the complicated part. A hereditarily countable set $a$ may be coded with a real by first computing the transitive closure of $\{a\}$, and then, since this is countable, by finding a relation $E$ on $\omega$ such that $\langle\operatorname{TC}(\{a\}), \in\rangle \cong\langle\omega, E\rangle$. We can then code the relation $E$ with a real in the usual manner (this coding technique is used extensively in section 8). This way of coding sets with reals works well with infinite time Turing machines. In particular, whether a real is a code is a $\Pi_{1}^{1}$ property; whether two codes code the same set is a $\Sigma_{1}^{1}$ property, as is whether one code codes a set which is an element of the set coded by another code. These elementary properties are therefore infinite time decidable. Indeed, the truth of any $\Delta_{0}$ set-theoretic property is decidable in the codes. Now, using the real $z$ which codes $\alpha$ to organize our construction, we can write a code for the set $L_{\alpha+1}$ by mimicking the construction of the $L$ hierarchy along the well order given by $z$. That is, we first use $z$ to reserve infinitely much room for each $\beta<\alpha$, and given the code for $L_{\beta}$ we then write down, from the definition, the code for $L_{\beta+1}$ in the space we had reserved. At limit stages, we simply write down the code for the union of sets whose codes we have already written down. Using the code for $L_{\alpha+1}$ that we have thus produced, we can check whether $z$ really is the least code in $L_{\alpha+1}$ for $\alpha$. And we can check whether $\alpha$ really is the least ordinal above $\delta$ such that $\alpha$ is countable in $L_{\alpha+1}$ (i.e. that $\alpha$ is $\beta$ ). If $z$ passes all of these tests, then $z$ must be $c$, otherwise it is not. So $\{c\}$ is decidable. It follows, for the second part of the theorem, that the set $\{(x, y) \mid y=c\}$ is decidable; this is the graph of the constant, total function $f(x)=c$. Thus, the graph of $f$ is decidable, but, since $c$ is not writable, $f$ is not computable.

We know how to code ordinals with reals. The previous proof, however, shows how to associate a unique such code to the ordinals which are countable in $L$. Namely, given any ordinal $\alpha$ which is countable in $L$, let $\beta$ be the least ordinal above $\alpha$ such that $L_{\beta+1}$ knows that $\beta$ is countable, and let $x$ be the least real, in the $L$ order, which codes $\beta$. The ordinal $\alpha$ is the $n^{\text {th }}$ element in the order given by $x$, for some $n$, and so $\alpha$ may be coded with the pair 
$\langle x, n\rangle$. This code is unique since $\alpha$ determines $\beta$, which determines $x$, and then $x$ and $\alpha$ determine $n$. Furthermore, the set of such codes, as we proved in the theorem, is a decidable set.

\section{Oracles}

Since infinite time Turing machines naturally compute functions on the reals, we are pushed towards two distinct kinds of oracles: individual reals and sets of reals. An individual real $x$ can be used as an oracle much as it is in the Turing machine context, by adding a special oracle tape on which $x$ is written out. This amounts, in effect, to having another input tape and using $x$ as an additional argument. Nevertheless, to highlight the oracle nature of the computations, if $p$ is a program using an oracle tape, then we will denote by $\varphi_{p}^{x}$ the resulting function which uses the real $x$ as an oracle. We refer to such functions as the infinite time $x$-computable functions.

Oracles, though, are properly the same type of object as decidable or semi-decidable sets; in the context of infinite time computations, this means that we want somehow to use a set of reals $A$ as an oracle. Clearly we cannot expect always to be able to write such an object out on a tape; but somehow we want the machine to be able to ask membership queries of $A$. Thus, we propose to add a special oracle tape, initially filled with zeros at the beginning of a computation, and to allow the machine during a computation to write on this tape and then, during a computation, by switching to a special oracle query state, to receive the answer Yes or No, on the cell beneath the head, accordingly as the real on the oracle tape is in $A$ or not. Thus, a machine with oracle $A$ is allowed to know if $y$ is a member of $A$, for any $y$ which it is able to write on the oracle tape. The machine can make as many such queries as it likes. We believe that this notion of oracle for sets of reals is natural and robust, resembling as it does the notion of constructibility from a predicate, as in the definition of $L[A]$. We can denote as usual by $\varphi_{p}^{A}(x)$ the resulting function computed by program $p$ with oracle $A$ on input $x$, and we refer to such functions as the infinite time $A$-computable functions, or, normally, just as the $A$-computable functions. If $x$ is a real, i.e. if $x \in 2^{\omega}$, let $A_{x}=\left\{\left(x\lceil n)^{\wedge}\langle 0,0, \ldots\rangle \mid n \in \omega\right\}\right.$ be the corresponding set oracle of finite approximations to $x$, concatenated with zeros. It is easy to see that a function is $x$-computable iff it is $A_{x}$-computable, because with the real oracle 
$x$ we can decide membership in $A_{x}$, and with the set oracle $A_{x}$, we can write $x$ on the tape. In this way real oracles can be thought of as a special case of set oracles. (Notice that, in view of the Lost Melody Theorem 4.9, being $x$-computable and being $\{x\}$-computable are very different things. In the former, we can see $x$ written out on the tape; in the latter, we are allowed only to know Yes or No whether any real we can produce is equal to $x$. But if $x$ is hard to produce, this is not helpful, since the answer will most likely be No. Indeed, this is the key significance of the Lost Melody Theorem 4.9.)

If $A$ and $B$ are two oracles, we will say that $A$ is infinite time computable from $B$, written $A \leq_{\infty} B$, when the characteristic function of $A$ is infinite time $B$-computable. This definition makes sense for both set and real oracles, if we think of reals as subsets of $\omega$. Since it is easy to verify that $\leq_{\infty}$ is transitive and reflexive, we also obtain the notion of infinite time degrees: $A \equiv_{\infty} B$ iff $A \leq_{\infty} B$ and $B \leq_{\infty} A$. This is an equivalence relation, and we denote the equivalence classes by $[A]_{\infty}$. Thus, for a real $x$, we have that $x \equiv_{\infty} A_{x}$. We will write $A<_{\infty} B$ when $A \leq_{\infty} B$ and $A \not \equiv_{\infty} B$. And of course we extend the notions of infinite time semi-decidability, clockability, and writability to the context of oracles in the obvious way, so that, for example, an ordinal $\alpha$ is $A$-clockable when there is program using oracle $A$ which halts on input 0 in exactly $\alpha$ many steps. Thus, a real $x$ is $A$-writable if and only if $A_{x} \leq_{\infty} A$ if and only if $x \leq_{\infty} A$. One last bit of notation: given two oracles $A$ and $B$, we write $A \oplus B$ to mean an oracle which codes, in some canonical manner, the information contained in $A$ and $B$. Thus, for example, $A \oplus B$ could be the reals resulting from adding a 0 digit to the front of every real in $A$, and a 1 to those in $B$. Clearly $A \oplus B$ is the least upper bound of $A$ and $B$ with respect to $\leq_{\infty}$.

We now define two jump operators, corresponding to the two halting problems. Suppose that $A$ is an oracle (either a set or a real oracle). The strong jump of $A$, denoted by $A^{\mathbf{v}}$, is simply the halting problem relativized to $A$. That is,

$$
A^{\boldsymbol{\nabla}}=H^{A}=\left\{(p, x) \mid \varphi_{p}^{A}(x) \downarrow\right\} .
$$

Secondly, the weak jump of $A$ is the set

$$
A^{\nabla}=A \oplus h^{A}=A \oplus\left\{p \mid \varphi_{p}^{A}(0) \downarrow\right\} .
$$

It may seem odd that we explicitly include the factor $A$ into $A^{\nabla}$, but the fact is that some sets of reals $A$ are sufficiently complex that they are not 
computable from any real; in particular, such sets $A$ are not computable from $h^{A}$ alone. Since we definitely want $A^{\nabla}$ to compute $A$, we are led to the definition above. When $A$ is a real, then it is not difficult to see that $A^{\nabla} \equiv_{\infty} h^{A}$, and consequently in this case we don't need to explicitly include $A$. Up to equivalence, $0^{\nabla}$ is just $h$, and $0^{\boldsymbol{\nabla}}$ is $H$. It is easy to see that any $A$-semi-decidable set is computable from $A^{\mathbf{\nabla}}$, and any $A$-semi-decidable real is computable from $A^{\nabla}$.

\section{Jump Theorem $5.1 A<_{\infty} A^{\nabla}<_{\infty} A^{\nabla}$.}

Proof: It is easy to see that $A \leq_{\infty} A^{\nabla} \leq_{\infty} A^{\mathbf{\nabla}}$, since $A^{\nabla}$ explicitly computes $A$, and $h^{A}$ appears as the $0^{\text {th }}$ slice of $A^{\mathbf{\nabla}}$. What remains is to prove the strict relations. The first argument is merely a relativization of Theorem 4.1. Relativizing that proof, we see that $h^{A}$ is not computable from $A$, and therefore, $A^{\nabla}$ is not computable from $A$, so $A<_{\infty} A^{\nabla}$.

Let us now prove that $A^{\nabla}<_{\infty} A^{\mathbf{v}}$. If not, then there is some program $q$ which computes $A^{\mathbf{\nabla}}$ from $A$ and $z=h^{A}$. That is,

$$
\varphi_{q}^{A \oplus z}(p, x)= \begin{cases}1, & \text { if } \varphi_{p}^{A}(x) \downarrow ; \\ 0, & \text { if } \varphi_{p}^{A}(x) \uparrow\end{cases}
$$

Let $f(r)$ be the program which halts on input $x$, using oracle $A$, exactly when $\varphi_{q}^{A \oplus x}(r, x)=0$. Thus, by the recursion theorem, there is a program $r$ such that $\varphi_{r}^{A}(x) \downarrow$ exactly when $\varphi_{q}^{A \oplus x}(r, x)=0$. But a special case of this is $\varphi_{r}^{A}(z) \downarrow \leftrightarrow \varphi_{q}^{A \oplus z}(r, z)=0$, which contradicts the assumption on $q$.

The argument just given actually establishes the following corollary.

No Reals Corollary 5.2 The set $A^{\boldsymbol{\nabla}}$ is not computable from $A \oplus z$ for any real $z$. In particular, 0 is not computable from any real.

Absorption Theorem 5.3 $A^{\nabla \mathbf{\nabla}} \equiv A^{\boldsymbol{\nabla}}$. Indeed, for any ordinal $\alpha$ which is $A^{\mathbf{v}}$-writable, $A^{\nabla^{(\alpha)} \mathbf{\nabla}}=A^{\mathbf{v}}$.

Proof: Let us prove the first equation first. Since $A \leq_{\infty} A^{\nabla}$, it is clear that $A^{\mathbf{\nabla}} \leq_{\infty} A^{\nabla \boldsymbol{\nabla}}$. It remains to show the converse relation. If $p$ is a program, let 
$f(p)$ be a program such that $\varphi_{f(p)}^{A}(x, y)=\varphi_{p}^{A \oplus y}(x)$. We may assume that $f$ is computable. Now simply compute

$$
\begin{gathered}
(p, x) \in A^{\nabla \boldsymbol{\nabla}}=\left(A \oplus h^{A}\right)^{\mathbf{\nabla}} \longleftrightarrow \varphi_{p}^{A \oplus h^{A}}(x) \downarrow \\
\longleftrightarrow \varphi_{f(p)}^{A}\left(x, h^{A}\right) \downarrow \longleftrightarrow\left(f(p),\left\langle x, h^{A}\right\rangle\right) \in A^{\boldsymbol{\nabla}} .
\end{gathered}
$$

Thus, $A^{\nabla \mathbf{\nabla}} \leq_{\infty} A^{\mathbf{\nabla}}$, and we are done.

Now, consider the more complicated equation $A^{\nabla^{(\alpha)} \mathbf{\nabla}}=A^{\mathbf{\nabla}}$, where $\alpha$ is coded by some real $z$ which is $A^{\boldsymbol{\nabla}}$ writable. We define the iterates $A^{\nabla^{(\alpha)}}$ with respect to $z$ by induction on $\alpha$, so that $A^{\nabla^{(\alpha)}}=A \oplus w_{\alpha}$ for some real $w_{\alpha}$. We begin of course with $w_{0}=0$, so that $A^{\nabla^{(0)}}=A \oplus 0$. At successor stages, we want

$$
A^{\nabla(\beta+1)}=\left(A^{\nabla(\beta)}\right)^{\nabla}=\left(A \oplus w_{\beta}\right) \oplus h^{A^{\nabla(\beta)}},
$$

and so we simply let $w_{\beta+1}=w_{\beta} \oplus h^{A^{\nabla^{(\beta)}}}$. At limit stages $\delta$, we let $w_{\delta}=$ $\oplus_{\beta<\delta} w_{\beta}$, using the real $z$ to organize the information. Now let us prove the theorem by induction on $\alpha$. Successor stages follow directly from the previous paragraph, since

$$
A^{\nabla(\beta+1)}=A^{\nabla(\beta)} \nabla \mathbf{\nabla}=A^{\nabla^{(\beta)} \mathbf{\nabla}}=A^{\mathbf{\nabla}},
$$

where the first equality follows from the definition of $A^{\nabla^{(\beta+1)}}$, the second from the previous absorption argument, and the third by the induction hypothesis. Now suppose $\delta$ is a limit ordinal, and the result holds for every $\beta<\delta$. Since $A^{\nabla^{(\delta)}}=A \oplus w_{\delta}$, where $w_{\delta}=\oplus_{\beta<\delta} w_{\beta}$ (using the real $z$ to organize the information), it follows that $w_{\delta}$ is computable from $A^{\boldsymbol{\nabla}}$ since the $w_{\beta}$ are uniformly computable from $A^{\boldsymbol{}}$. We are really just iterating the argument of the previous paragraph along the order coded by the real $z$. So, to finish the theorem, we just have to argue that $\left(A \oplus w_{\delta}\right)$ is computable from $A^{\mathbf{\nabla}}$. To see why this is so, let $q$ be the program such that $\varphi_{q}^{A}(y, p, x)=\varphi_{p}^{A \oplus y}(x)$. Thus, $(p, x) \in\left(A \oplus w_{\delta}\right)^{\boldsymbol{\nabla}} \leftrightarrow\left(q,\left\langle w_{\delta}, p, x\right\rangle\right) \in A^{\boldsymbol{\nabla}}$. And with $A^{\boldsymbol{\nabla}}$ we can compute this latter property, so the proof is complete.

The next theorem shows that the infinite time jump operators $\nabla$ and $\mathbf{v}$ jump much higher than the Turing jump, even when the Turing jump is iterated an enormous number of times.

Jump Closure Theorem 5.4 Every infinite time degree is closed under the Turing jump operator. Indeed, for any real $x$ and any writable ordinal $\alpha$, the $\alpha^{\text {th }}$ Turing jump of $x$ is still infinite time equivalent to $x$. 
Proof: The Turing jump of a real $x$ is defined to be $x^{\prime}=\left\{e \in \mathbb{N}:\{e\}^{x}(e) \downarrow\right\}$, where $\{e\}^{x}(e)$ denotes the Turing machine computation of program $e$ on input $e$ with oracle $x$. The $\alpha^{\text {th }}$ jump of $x$, relative to a real $z$ coding a relation on $\omega$ of length $\alpha$, is the subset of the plane $\omega \times \omega$ whose $(\beta+1)^{\text {th }}$ column is the Turing jump of the $\beta^{\text {th }}$ column, and at limits a sum is taken using the canonical order given by $z$. The point is that the Turing machine halting problem is infinite time computable in $\omega$ many steps, and so an infinite time machine can systematically compute the iterates of the Turing jumps by simply solving the Turing machine halting problem for the various oracles, and using the coding of $\alpha$ by the real $z$ to organize the information.

Theorem 5.5 For any oracle $A, A^{\nabla} \equiv_{\infty} H_{\gamma^{A}}^{A}$, where $\gamma^{A}$ is the supremum of the A-clockable ordinals.

Proof: First, let us argue that $H_{\gamma^{A}}^{A} \leq_{\infty} A^{\nabla}$. For this, consider the algorithm which on input $(p, x)$ first consults $A^{\nabla}$ to find which programs $r$ halt with oracle $A$ on input 0 . Then, the algorithm simulates the computation $\varphi_{r}^{A}(0)$ for all these $r$, at the same time simulating the computation $\varphi_{p}^{A}(x)$. The computations $\varphi_{r}^{A}(0)$ act as a clock which will run out at $\gamma^{A}$. If the computation of $\varphi_{p}^{A}(x)$ finishes before all the clocks have run out, then $(p, x)$ is in $H_{\gamma^{A}}^{A}$, and we output Yes. Otherwise, the clocks all run out first, and we output No.

Second, we will argue that $A^{\nabla} \leq_{\infty} H_{\gamma^{A}}^{A}$. Certainly $A$ is computable from $H_{\gamma A}^{A}$, by considering the algorithm which halts on elements of $A$ and otherwise does not halt (using $A$ as an oracle). So it remains only to show that $h^{A}$ is computable from $H_{\gamma^{A}}^{A}$. But this is clear, since a program $p$ with oracle $A$ on input 0 will halt before $\gamma^{A}$ if it halts at all, because the length of the computation will be an $A$-clockable ordinal. So $p \in h^{A} \longleftrightarrow(p, 0) \in H_{\gamma^{A}}^{A}$. Thus, both $A$ and $h^{A}$ are computable from $H_{\gamma^{A}}^{A}$, and so $A^{\nabla} \leq_{\infty} H_{\gamma^{A}}^{A}$, as desired.

Theorem $5.6 \Delta_{2}^{1}$ is closed under the jump operators $\nabla$ and $\mathbf{}$.

Proof: We simply have to check that if $A$ is $\Delta_{2}^{1}$, then so is $A^{\mathbf{\nabla}}$. But $(p, x)$ is in $A^{\mathbf{v}}$ exactly when there is a real coding a well-ordered sequence of snapshots 
according to program $p$ on input $x$ with oracle $A$ which shows the computation to halt. Let us say this in more detail: $(p, x)$ is in $A^{\mathbf{\nabla}}$ exactly when there is a real $z$ which codes a well-ordered sequence of snapshots such that first, the initial snapshot is the starting configuration of $\varphi_{p}^{A}(x)$; second, at every step of this sequence either an ordinary computation was performed by $p$ to obtain the next snapshot, or, if $z$ indicates that an oracle query was made to which the answer $z$ provided was Yes, then there is a real $y$ which is the real the query was made about, and this real is in $A$, or, if $z$ indicates that an oracle query was made to which the answer $z$ provided was No, then there is a real $y$ which is the real that the query was made about, and this real is not in $A$; third, the snapshots at limit stages are obtained from the previous snapshots according to the lim sup rule; and fourth, that the final snapshot shows the computation to have halted. Thus, $A^{\mathbf{v}}$ is $\Sigma_{2}^{1}$. Similarly, $(p, x)$ is in $A^{\boldsymbol{\nabla}}$ exactly when every real coding a well-ordered sequence of snapshots according to $p$ on input $x$ with oracle $A$ which is settled shows the computation to halt. This is $\Pi_{2}^{1}$, and so $A^{\mathbf{\nabla}}$ is $\Delta_{2}^{1}$, as desired.

Let us now analyze the complexity of the infinite time degree relation. Recall that $x \leq_{\infty} y$ when $x$ is infinite time computable from $y$.

Theorem 5.7 The relation $x \leq_{\infty} y$ is semi-decidable but not decidable.

Proof: Notice that $x \leq_{\infty} y$ exactly when there is a program $p$ such that $\varphi_{p}^{y}(0)=x$. So we can simply try them all out. That is, on input $x$ and $y$, simultaneously, for each program $p$, simulate the computation of $\varphi_{p}^{y}(0)$, and, when and if the simulations halt, check if the output is $x$. If so, output Yes. This algorithm gives the affirmative answers to $x \leq_{\infty} y$, and therefore that relation is infinite time semi-decidable.

Now let us argue that it is not decidable. First note that there are accidentally writable reals that are not decidable, since $0^{\nabla}$ is such a real. Assume towards a contradiction that $\leq_{\infty}$ is decidable, and consider the following supertask algorithm: for each program $p$ simulate the computation of program $p$ on input 0 , and after each step of the simulated computation, check if the real on the simulated tape is decidable (this is possible by our assumption). If a nondecidable real is found, then write it on the output tape and halt. Since there are accidentally writable reals which are not decidable, this algorithm will halt with a nondecidable real written on the tape. But this is a 
contradiction, since such a real cannot be writable.

For the next theorem, denote by $\lambda^{A}$ the supremum of the $A$-writable ordinals, which is also the order-type of the $A$-clockable ordinals. And, as before, let $\gamma^{A}$ be the supremum of the $A$-clockable ordinals.

Theorem 5.8 The ordinal $\lambda^{A}$ is $A^{\nabla}$-writable in $\gamma^{A}$ many steps, and it is eventually $A$-writable, but not $A$-writable. The eventually $A$-writable ordinals are the same as the eventually $A^{\nabla}$-writable ordinals.

Proof: The first part of this theorem follows by simply relativizing the argument of 3.8 to the context of the oracle $A$. This algorithm involves computing the relation $p \triangleleft q$ iff the computation $\varphi_{p}^{A}(0)$ halts before $\varphi_{q}^{A}(0)$. The relation has rank $\lambda^{A}$, and the natural way to write it takes $\gamma^{A}$ many steps (one must consult $A^{\nabla}$, asking which computations $\varphi_{p}^{A}(0)$ will halt, in order to know that the computation has finished). By omitting this last part, about consulting $A^{\nabla}$ to know whether the algorithm has finished, it follows that $\lambda^{A}$ is eventually $A$-writable. And by relativizing Theorem 3.8 again, we see that $\lambda^{A}$ is not $A$-writable.

Since $h^{A}$ is eventually $A$-writable, any ordinal which is eventually $A^{\nabla}$ writable is actually eventually $A$-writable, because with only $A$ as an oracle, we can compute approximations to $h^{A}$, and then, with these approximations, run the program which eventually writes an ordinal from $A^{\nabla}$. Eventually, this algorithm will have the true $h^{A}$ written, and the correct ordinal will be eventually written.

\section{The Structure of Infinite Time Degrees}

In this section we would like to give an introductory analysis of the structure of the infinite time degrees. Initially, one might hope to mimic many of the results from classical recursion theory, perhaps even giving some priority arguments, but this hope must be tempered by the realization that one cannot preserve a computation by fixing only finitely much of an oracle; after all, the computation to be preserved may have used the entire oracle. Nevertheless, we have managed to prove a few facts, and content ourselves to leave the vast bulk of results for others to prove. 
Let us first prove some elementary facts.

Theorem 6.1 There are incomparable infinite time degrees in the reals.

Proof: This proof also works in the Turing degree case. Assume that the infinite time degrees are linearly ordered. Since every initial segment of this order is countable - there being only countably many programs - it follows that there are at most $\aleph_{1}$ many degrees, and consequently the Continuum Hypothesis holds. But the Continuum Hypothesis fails in a forcing extension. Consequently, in such extensions there are incomparable degrees. Furthermore, by Theorem 5.7, the assertion that there are incomparable degrees is a $\Sigma_{2}^{1}$ assertion, and thus, by the Shoenfield absoluteness theorem, it is absolute to any forcing extension. Hence, there must have been infinite time incomparable reals originally.

The previous theorem can be improved to the following.

Antichain Theorem 6.2 There is a countable sequence of reals, no one of which is infinite time computable from the others.

Proof: Since it is well known that no real in a sequence of mutually generic Cohen reals can be constructed from the rest of the sequence, the assertion is true after forcing to add $\omega$ many Cohen reals. Furthermore, the assertion is $\Sigma_{2}^{1}$. Hence, by the Schoenfield absoluteness theorem, it must have been true originally.

The next two theorems are straightforward adaptations of the classical arguments (see, e.g., Soare).

Corollary 6.3 Any countable partial order embeds into the real infinite time degrees.

Proof: Suppose $\langle\omega, \preceq\rangle$ is a partial order on $\omega$, and $\left\langle z_{n} \mid n \in \omega\right\rangle$ is a sequence of reals, no one of which is computable from the others and $\langle\omega, \preceq\rangle$. It is straightforward to verify that the map $n \mapsto \oplus\left\{z_{i} \mid i \preceq n\right\}$ embeds the partial order into the real infinite time degrees. 
Theorem 6.4 In the infinite time degrees, a simple set exists. That is, there is a semi-decidable set $S$ whose complement is infinite but contains no infinite semi-decidable set.

Proof: For this argument, we can simply mimic Post's proof in the classical argument. Namely, for each program $p$, start computing $\varphi_{p}(n)$ for all the values of $n \in \omega$, and let $f(p)$ be the first $n>2 p$ for which $\varphi_{p}(n) \downarrow$. Let $s=$ ran $(f)$. Thus, $s$ is a subset of $\omega$, and it is clearly semi-decidable. Also, the complement $\omega \backslash s$ is infinite since $s$ contains at most $p$ many elements below $2 p$. But $s$ meets every infinite semi-decidable subset of $\omega$, so the complement $\omega \backslash s$ contains no infinite semi-decidable set. To construct a set of reals with the same property, simply observe that we can regard $\omega \subset \mathbb{R}$, and then let $S=(\mathbb{R} \backslash \omega) \cup s$. Thus, $S \subset \mathbb{R}$ is semi-decidable with an infinite complement in $\mathbb{R}$ which contains no infinite semi-decidable set.

\section{Questions $^{2} 6.5$}

Is there a semi-decidable set of reals $S$ whose complement has size the continuum but contains no infinite semi-decidable set?

Are there incomparable degrees below $0^{\nabla}$ ? Below 0 ?

Are there any noncomputable degrees below $0^{\nabla}$ ?

We would like in the next few theorems to understand the relationship between the degrees represented by a real and the degrees which are represented only by sets of reals.

Theorem 6.6 Any countable set of reals is infinite time computable from a real.

Proof: Suppose that $A=\left\{z_{n} \mid n \in \omega\right\}$. Let $z$ canonically code the sequence $\left\langle z_{n} \mid n \in \omega\right\rangle$. Clearly $A$ is computable from $z$, since an infinite time Turing machine can perform the decoding.

\footnotetext{
${ }^{2}$ In a forthcoming paper, we provide the intriguing answer to all but the first of these questions, by showing that while there are no reals between 0 and $0^{\nabla}$, there are incomparable sets of reals between 0 and $0^{\nabla}$. This latter fact is proved by adapting the classical priority argument technique to the supertask context.
} 
Theorem 6.7 Most oracles $A \subset \mathbb{R}$ are not computable from a real.

Proof: This is a simple counting argument. There are only $2^{\omega}$ many reals, and each of them computes at most countably many sets, so there are only $2^{\omega}$ many sets which are computable from a real, but there are $2^{2^{\omega}}$ many sets of reals.

The interesting thing, however, is that one can also ensure that there are no noncomputable reals below an oracle.

Theorem 6.8 There is a countable semi-decidable set $W$ such that any larger set $B \supseteq W$ computes no noncomputable reals. In fact, the set $W$ can be taken to be the set of accidentally writable reals.

Proof: Let $W$ be the set of accidentally writable reals. This set is certainly countable and semi-decidable. Moreover, it includes, and in fact is equal to, the set of reals for which an oracle query is made during the computation of $\varphi_{p}^{\mathbb{R}}(0)$, for any program $p$. Notice that when $\mathbb{R}$ is the oracle, queries are always answered Yes. But $W$ will also answer Yes to all those queries, since if on input 0 a real is written on the tape and a query made about it, it must have been an accidentally writable real. Consequently, we have $\varphi_{p}^{W}(0)=\varphi_{p}^{\mathbb{R}}(0)$, for any program $p$ (meaning that if one converges, then both converge to the same answer). Furthermore, if $W \subset B$, then similarly $\varphi_{p}^{B}(0)=\varphi_{p}^{\mathbb{R}}(0)$. Now if $z$ is computable from $B$, it must be that $z=\varphi_{p}^{B}(0)$ for some program $p$, and consequently $z=\varphi_{p}^{\mathbb{R}}(0)$ also. Thus, $z$ is computable from $\mathbb{R}$, and hence it is computable.

Corollary 6.9 There is a set B of reals which neither computes any noncomputable real, nor is computable from any real. 
Proof: Simply combine the two previous arguments. There are $2^{2^{\omega}}$ many sets $B$ which contain $W$, but only $2^{\omega}$ many of them are computable from a real, so there must be some $B$ containing $W$ which is not computable from a real. Since it contains $W$, it also fails to compute any noncomputable real. $\square$

Theorem 6.10 For every real $x$ there is a set $A$ which computes the same reals as $x$ but which itself is not computable from any real.

Proof: Fix the real $x$. Let $Q_{x}$ be the set of reals for which a query is made during the computations of the form $\varphi_{p}^{\mathbb{R}}(x)$. Since the oracle is $\mathbb{R}$, such queries are always answered Yes. If $B$ is a set such that $Q_{x} \subseteq B \subseteq \mathbb{R}$, then $B$ will also give the answer Yes to all such queries, and so $\varphi_{p}^{B}(x)=\varphi_{p}^{\mathbb{R}}(x)$ for any program $p$. Thus, the reals below $B \oplus x$ are exactly those below $x$. By the counting argument, we may find a $B$ which is not computable from any real. Let $A=B \oplus x$ for such a $B$. Then the reals computable from $A$ are exactly the reals computable from $x$, and $A$ is not computable from any real. $\square$

Theorem 6.11 For every set $A$ there is a countable set $B \subset A$ which is semi-decidable in $A$ and computes the same reals as $A$. 
Proof: Let $B$ be the set of reals in $A$ for which a query is made during a computation of the form $\varphi_{p}^{A}(0)$. Thus, for any $\operatorname{program} p$, we have $\varphi_{p}^{B}(0)=\varphi_{p}^{A}(0)$, since $B$ answers Yes whenever $A$ does for such computations. It follows that $A$ and $B$ compute the same reals. The set $B$ is countable since there are only countably many programs $p$, and each computation $\varphi_{p}^{A}(0)$ mentions only countably many reals. Clearly $B$ is semi-decidable in $A$, since one simply simulates the computations $\varphi_{p}^{A}(0)$, and says Yes to any real in $A$ which appears along the way.

Jump Iteration Theorem 6.12 If a real $z$ is computable from $0 \mathbf{\nabla}$, then so is $z^{\nabla}$. Indeed, we may iterate the jump operator $\nabla$ many times: if $\alpha$ is $0^{\nabla}$-writable, then $z^{\nabla^{(\alpha)}} \leq_{\infty} 0^{\nabla}$.

Proof: Suppose $z=\varphi_{r}^{0 \nabla}(0)$. Let $\varphi_{q}(p, x)=\varphi_{p}^{x}(0)$. Thus, $p \in z^{\nabla} \leftrightarrow$ $\varphi_{p}^{z}(0) \downarrow \leftrightarrow \varphi_{q}(p, z) \downarrow \leftrightarrow\left(q,\left\langle p, \varphi_{r}^{0^{\boldsymbol{\nabla}}}(0)\right\rangle\right) \in 0^{\boldsymbol{\nabla}}$. So $z^{\nabla}$ is computable from $0^{\boldsymbol{\nabla}}$. Suppose now that $\alpha$ is coded by the $0^{\nabla}$-writable real $y$, and we want to iterate the jump $\alpha$ many times. We use the real $y$ to organize the scratch tape into $\alpha$ many rows, putting the jump of each row into the next row (according to the order given by $y$ ). At limit stages, we write the real coding all of the earlier reals according to the organization given by $y$. When we have finished, we have a real coding $z^{\nabla^{(\alpha)}}$, as desired.

Low Theorem 6.13 Every real below $0^{\mathbf{\nabla}}$ is low. That is, if $z<_{\infty} 0^{\mathbf{\nabla}}$, then $z^{\mathbf{\nabla}} \equiv_{\infty} 0 \mathbf{\nabla}$. And this may be relativized to any oracle: every real below $A^{\mathbf{\nabla}}$ is A-low, in the sense that if $z<_{\infty} A^{\mathbf{\nabla}}$, then $z^{\mathbf{\nabla}} \leq_{\infty} A^{\mathbf{\nabla}}$.

Proof: Certainly $0^{\boldsymbol{\nabla}} \leq_{\infty} z^{\boldsymbol{\nabla}}$, so we must only show the other direction. Suppose $z=\varphi_{r}^{0 \nabla}(0)$. Let $\varphi_{q}(p, x, y)=\varphi_{p}^{y}(x)$. Thus,

$$
\begin{gathered}
(p, x) \in z^{\boldsymbol{\nabla}} \longleftrightarrow \varphi_{p}^{z}(x) \downarrow \longleftrightarrow \varphi_{q}(p, x, z) \downarrow \\
\longleftrightarrow(q,\langle p, x, z\rangle) \in 0^{\mathbf{\nabla}} \longleftrightarrow\left(q,\left\langle p, x, \varphi_{r}^{0 \boldsymbol{\nabla}}(0)\right\rangle\right) \in 0^{\boldsymbol{\nabla}} .
\end{gathered}
$$

Thus, $z^{\boldsymbol{\nabla}}$ is computable from $0 \mathbf{\nabla}$ and we are done. The same argument works for any oracle $A$. 
Eventual Jump Theorem 6.14 The class of eventually writable reals is closed under the jump operator $\nabla$. Indeed, if $z$ is an eventually writable real, and $\alpha$ is an eventually writable ordinal, then the $\alpha^{\text {th }}$ iterate $z^{\nabla^{(\alpha)}}$ of the jump operator $\nabla$ is still eventually writable.

Proof: Suppose that $z$ is eventually written by program $p$. Consider now the supertask algorithm that simulates $p$ on a portion of the scratch tape. We regard the various reals that appear on the output tape of the simulation as approximations to $z$. For every such approximation $w$, we begin the computation to write $w^{\nabla}$ on the output tape, while continuing the simulation of $p$ on the scratch tape. If we find that the approximation $w$ changes at some point, then we disregard our previous attempts to compute $w^{\nabla}$, and start fresh with the new approximation. Eventually, the real $z$ will appear as its own approximation, never subsequently to be changed, and we will eventually write $z^{\nabla}$ on the output tape, never subsequently to erase it.

A similar argument shows that $z^{\nabla^{(\alpha)}}$ is also eventually writable. For this argument, the algorithm also computes approximations to $\alpha$, and for each such approximation, it uses the previous technique to compute the $\nabla$ iterates of $z$. Eventually, the algorithm will have the true approximation to $z$ and the true approximation to $\alpha$, and after a very long time, the true approximation to $z^{\nabla^{(\alpha)}}$. So $z^{\nabla^{(\alpha)}}$ is eventually writable.

In the next theorem, we regard a real as semi-decidable when there is a supertask algorithm which gives the affirmative answers to queries about the digits of the real.

\section{Implication Theorem 6.15 1. Every writable real is semi-decidable;}

2. every semi-decidable real is eventually writable;

3. every eventually writable real is accidentally writable;

4. every eventually writable real is computable from 0 ;

5. and none of these implications is reversible.

Proof: Clearly every writable real is semi-decidable. One simply runs the program which writes the real, consults this real, and answers Yes appropriately. Also, every semi-decidable real is eventually writable. One simply simulates simultaneously for each natural number $n$ the program to semi-decide 
whether $n$ is in the real. Eventually all the Yes answers will be obtained and the real will be written on the output tape, even while it is searching for more Yes answers, not realizing that it has already found them all. And obviously every eventually writable real is accidentally writable. It remains to check that every eventually writable real is computable from $0^{\boldsymbol{\gamma}}$. Suppose $x$ is eventually written by the program $p$ (we might write in this case $\left.\varphi_{p}(0) \uparrow=x\right)$. Consider the algorithm which simulates the computation of $\varphi_{p}(0)$. At any stage of this simulation, we can ask the oracle 0 whether the algorithm which would search for this snapshot to repeat again would ever find that it does. When the oracle answers Yes, we know we have reached the looping part of the simulation, and we can halt, knowing that $x$ must be written on the output tape.

Now we must prove that none of the implications is reversible. The real $0^{\nabla}$ is semi-decidable but not writable. By the previous theorem, the real $0^{\nabla \nabla}$ is eventually writable but not semi-decidable, since it is not computable from $0^{\nabla}$. Next, the hard part, we must prove that there is an accidentally writable real which is not eventually writable. Consider the algorithm which simulates the computation of $\varphi_{p}(0)$ for every program $p$, and, at every step of these computations, writes on the output tape a real which diagonalizes against the reals on the simulated output tapes. Thus, for every simulated step, we write a real on the output tape which differs from every real appearing on the simulated output tapes. Eventually, the programs $p$ which produce eventually writable reals have stabilized in the sense that they have reached the stage where the real is written on their output tape, not to be subsequently changed, and our algorithm then writes a real which is different from all of them (and also different from some other irrelevant reals). Thus, our algorithm writes a real which is not eventually writable. Finally, we must show that there is a real which is computable from $0^{\mathbf{v}}$ which is not eventually writable. Let us define that a computation $\varphi_{p}(x)$ stabilizes if it either halts or eventually writes a real on its output tape which is not subsequently changed. Let $S$ be the set of programs $p$ which stabilize on input 0 . The previous diagonal argument shows that $S$ cannot be eventually writable, since we could have just diagonalized against the reals resulting from programs in $S$ to arrive at the same contradiction there. But nevertheless, the set $S$ is computable from $0^{\boldsymbol{v}}$, as we will now show. Consider the algorithm which simulates the computation of all $\varphi_{p}(0)$. Eventually, all the programs which will stabilize have stabilized, and only then will the simulation go into an 
infinite repeating loop. At any stage of the simulation, we can ask the oracle 0 whether we have reached a snapshot yet which will be repeated later. Since $0^{\boldsymbol{\nabla}}$ can answer such questions, we will know whether we have reached the infinite repeating loop. After having reached this loop, we run through the loop once, checking which of the computations $\varphi_{p}(0)$ change their output tape. The ones that do not are exactly the elements of $S$. So $S$ is computable from 0 .

It is possible to show also that $S$ is accidentally writable. We do not know, however, the answer to the following question.

Question 6.16 Is every accidentally writable real computable from 0 ? Or vice versa?

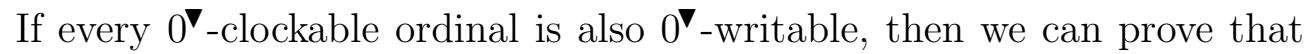
there is a real computable from $0 \nabla$ which is not accidentally writable.

\section{$7 \quad$ A new pointclass below $\triangle_{2}^{1}$}

In this section we will analyze a boldface version, if you will, of the decidable sets. Namely, we define that a set of reals $A$ is decidable from a real when there is a real $z$ with respect to which $A$ is decidable. These sets form a new natural pointclass below $\Delta_{2}^{1}$. The jump operator will stratify the $\Delta_{2}^{1}$ sets, and indeed all sets of reals, into a fine hierarchy, ordered by $\leq_{\infty}$.

Decidable Pointclass Theorem 7.1 The class of sets which are decidable from a real is a $\sigma$-algebra, and is closed under Suslin's operation $\mathcal{A}$. Hence, they include the $C$-sets of Selivanovski. But this inclusion is proper, for there is a decidable set which is not a $C$-set.

Proof: The C-sets of Selivanovski are defined to be those in the smallest $\sigma$ algebra containing the Borel sets which is closed under Suslin's operation $\mathcal{A}$. The sets decidable from a real are clearly closed under complement. For the first part of the theorem, we need to prove they are closed under countable union. Suppose that for each natural number $n$, the set $A_{n}$ is decidable by $\operatorname{program} p_{n}$ from real $z_{n}$. Let $z$ be a real which canonically codes $\left\langle z_{n} \mid n \in \omega\right\rangle$ and $\left\langle p_{n} \mid n \in \omega\right\rangle$. Consider the supertask algorithm which on input $x$ uses $z$ 
as an oracle to systematically check whether $x \in A_{n}$. The algorithm simply simulates $p_{n}$ with oracle $z_{n}$ on input $x$, and gets the answer yes or no whether $x \in A_{n}$. After our algorithm has finished the simulations, let it output yes or no accordingly if $x$ was in any of the sets $A_{n}$. Thus, $\cup_{n} A_{n}$ is decidable from $z$, and so the class of sets decidable from reals is a $\sigma$-algebra.

For the second part, recall that Suslin's operation $\mathcal{A}$ is defined on a family of sets $\left\langle A_{s} \mid s \in \omega^{<\omega}\right\rangle$ to be the set of $x$ such that there is a $y \in \omega^{\omega}$ such that $x \in A_{y\lceil n}$ for every natural number $n$. Thus, $x \in \mathcal{A}\left(\left\langle A_{s} \mid s \in \omega^{<\omega}\right\rangle\right)$ exactly when $\left\{s \mid x \in A_{s}\right\}$ has an infinite branch. By intersecting the sets with the ones preceding them, it suffices to consider only the case when $s \subset t \rightarrow A_{t} \subset A_{s}$, so that $\left\{s \mid x \in A_{s}\right\}$ is a tree. So, suppose $A_{s}$ is decidable from $z_{s}$ by the program $p_{s}$ for each $s \in \omega^{<\omega}$. Let $z$ be a real which canonically codes the other reals $\left\langle z_{s} \mid s \in \omega^{<\omega}\right\rangle$ as well as the programs $\left\langle p_{s} \mid s \in \omega^{<\omega}\right\rangle$. Now suppose we are given $x$, and we wish to decide if $x \in \mathcal{A}\left(\left\langle A_{s} \mid s \in \omega^{<\omega}\right\rangle\right)$. Using $z$ as an oracle, we can simulate for each $s$ the program $p_{s}$ with oracle $z_{s}$ on input $x$, and write out the set $\left\{s \mid x \in A_{s}\right\}$. We can assume this is a tree. In $\omega$ many steps, we can write out the relation coding the Kleene-Brouwer order on this tree, and then use the count-through algorithm to determine if this order is a well order. Since this occurs exactly when the tree has no branches, this algorithm can decide whether $x \in \mathcal{A}\left(\left\langle A_{s} \mid s \in \omega^{<\omega}\right\rangle\right)$. So the class of sets decidable from a real is closed under Suslin's operation $\mathcal{A}$. Thus, every $C$-set is decidable from a real.

Lastly, we would like to show that this inclusion is proper. There is a natural way to code C-sets - one just labels each node in a countable wellfounded tree with instructions for taking the union, complement, or operation $\mathcal{A}$ of the children of that node. The leaves of the tree are labeled with basic open sets. The set coded by such a code is obtained by simply working up the tree, assigning a set to each node according to the instructions on the labels. The set assigned to the top node is the desired set. And every C-set is coded by such a code. The labeled tree itself can easily be coded by a real, and the set of reals coding $\mathrm{C}$-set codes is $\Pi_{1}^{1}$, since the only complicated part is that the tree must be well-founded. Thus, the set of $\mathrm{C}$-set codes is decidable. If $w$ is a C-set code, let $A_{w}$ be the C-set which $w$ codes. Let us argue that the relation $x \in A_{w}$ is infinite time decidable. First, we already argued that we can decide if $w$ is in fact a C-set code. If it is, then we can systematically decide whether $x \in A_{y}$ for each code $y$ which appears as a node in the tree 
coded by $w$. Thus, at the leaves of the tree, we decide if $x$ is in the basic open set at that node. If a node instructs us to take a complement at that node, then we flip the previous answer to its opposite. If a node instructs us to take a union of the previous nodes, then we search to see if $x$ is in any of the previous nodes, and write the answer accordingly. Lastly, if a node instructs us to apply Suslin's operation $\mathcal{A}$ to the previous nodes, then we have to write down the Kleene-Brouwer order on the 'tree' of finite sequences coded in the children whose $\mathrm{C}$-sets contain our given real $x$. If this order is a well-order then we write No, otherwise Yes, on the node labeled $\mathcal{A}$. At the end of this algorithm, the top node has been labeled according to whether $x \in A_{w}$ or not, and we output the answer. It follows by this argument that the set $D=\left\{w \mid w \notin A_{w}\right\}$ is decidable. Furthermore, it is easy to see that it is not a C-set, because it cannot be equal to $A_{w}$ for any code $w$. So the theorem is proved.

Remark 7.2 The previous theorem has a lightface analog. Namely, the class of decidable sets is effectively a $\sigma$-algebra, in the sense that it is closed under complements and if the sets $\left\langle A_{n} \mid n \in \omega\right\rangle$ are uniformly decidable, then so is their union; and the class of decidable sets is closed under effective applications of $\mathcal{A}$ in the sense that if $\left\langle A_{s} \mid s \in \omega^{<\omega}\right\rangle$ is uniformly decidable, then so is $\mathcal{A}\left(\left\langle A_{s} \mid s \in \omega^{<\omega}\right\rangle\right)$. 
The previous theorems identify a new hierarchy of pointclasses between the C-sets and the $\Delta_{2}^{1}$ sets, as illustrated in the previous diagram. One moves up the hierarchy by applying the jump operator. The next theorem tells us some more descriptive-set-theoretic information about the class of semidecidable sets. For those unfamiliar with some descriptive set theoretic terms, [Mos provides an excellent and full description of such concepts as norms (p.69), the prewellordering property (p. 200), and Spector point classes (p. 207).

Semi-decidable Norm Theorem 7.3 Every semi-decidable set admits a semi-decidable norm. Thus, the class of semi-decidable sets has the prewellordering property, and is a Spector pointclass.

Proof: Suppose $A$ is semi-decidable by some program $p$ (for definiteness, assume $p$ is the least such program). Thus, $x \in A$ if and only if $\varphi_{p}(x) \downarrow$ 
(and in this case the value is 1 ). Let $\rho(x)$ be the number of steps that the computation $\varphi_{p}(x)$ takes to halt, if it does halt. This is obviously a norm on $A$. What we have to show is that the two relations

$$
\left.x \leq_{\rho} y \leftrightarrow x \in A \wedge[y \notin A \vee \rho(x) \leq \rho(y))\right]
$$

and

$$
x<_{\rho} y \leftrightarrow x \in A \wedge[y \notin A \vee \rho(x)<\rho(y)] .
$$

are both semi-decidable. The first relation $x \leq_{\rho} y$ holds exactly when the computation of $\varphi_{p}(x)$ halts before or simultaneously with $\varphi_{p}(y)$, and this is clearly semi-decidable, since we can simply simulate both computations, checking to see if $\varphi_{p}(x)$ halts in time. The second relation $x<_{\rho} y$ holds exactly when the computation $\varphi_{p}(x)$ converges and halts strictly before $\varphi_{p}(y)$. This is also semi-decidable by a similar algorithm.

The class of semi-decidable sets is clearly a $\Sigma$-pointclass with the substitution property, closed under $\forall^{\omega}, \omega$-parameterized by the programs, and, by the argument just given, normed. Thus, it is a Spector pointclass.

\section{Admissibility}

In this section we would like to explore the connections between infinite time computability and admissible set theory. A transitive collection of sets is admissible when it is a model of the following set theoretic axioms: pairing, union, infinity, Cartesian product, $\Delta_{0}$-comprehension, and $\Delta_{0}$-collection. We will refer to these axioms as the Kripke-Platek axioms. We refer the reader to [Bar] for an excellent account of admissibility, and content ourselves here to say that the KP axioms form a particularly important fragment of set theory. Though weak, this fragment is sufficiently strong to carry out many settheoretic constructions, such as that of Gödel's $L$. An ordinal $\alpha$ is admissible when $L_{\alpha}$ is an admissible set.

Our infinite time Turing machines of course deal only with reals, but we can quite easily use reals to code hereditarily-countable sets. Specifically, given a hereditarily-countable set $a$, one enumerates $T C(\{a\})=\left\{a_{n} \mid\right.$ $n \in \omega\}$, and then defines a relation $i E j \leftrightarrow a_{i} \in a_{j}$. Thus, $\langle\omega, E\rangle \cong$ $\langle T C(\{a\}), \in\rangle$, and so from $E$ we can recover $T C(\{a\})$, and hence also $a$ 
(since $\{a\}$ is the unique member of $T C(\{a\})$ which is not a member of any other member). The relation $E$ can be coded with a real in the usual manner. For the rest of this section, let us assume complete familiarity with such coding techniques, and move on now to prove the admissibility of the various collections of sets to which the machines have access.

Theorem 8.1 The class of sets coded by writable reals is admissible.

Proof: The first thing to notice is that the set of reals which are codes for sets is $\Pi_{1}^{1}$ and therefore decidable. To determine whether two codes actually code the same set is $\Sigma_{1}^{1}$ (since this is true if and only if there is an isomorphism between the indexed sets) and therefore also decidable, as is determining whether codes $x$ and $y$ code sets such that $A_{x} \in A_{y}$. Thus, it is easy to see that $\Delta_{0}$ facts about the sets coded by reals are decidable from the codes. Furthermore, from a code $z$ for the set $A_{z}$, a code can be generated for any element $a \in A_{z}$, knowing merely which natural number $n$ represents $a$ in the code $z$. The class of sets coded by writable reals is clearly closed under union, pairing, and difference. And it satisfies $\Delta_{0}$-comprehension because from a code $z$ for a set $A_{z}$, and a $\Delta_{0}$-formula $\psi$, we can systematically compute a code for the set $\left\{a \in A_{z} \mid \psi(a)\right\}$ by simply computing whether the formula $\psi$ holds separately for each member of the set, and then putting all desired elements together into a code. Finally, we will prove that $\Delta_{0^{-}}$ collection holds. So suppose $z$, coding the set $A_{z}$, is writable, and for every $a \in A_{z}$ there is a set $b$ coded by a writable real such that $\psi(a, b)$ holds, where $\psi$ is a $\Delta_{0}$ formula. What we need to find is a set $B$ which is coded by a writable real such that for every $a \in A_{z}$ there is a witness $b \in B$ such that $\psi(a, b)$. Let us now show there is such a $B$. Fix $z$, and consider the supertask algorithm which first writes $z$ on a portion of the scratch tape. Now, we will slowly write down the code for $B$, by searching for witnesses. Every element of $A_{z}$ is indexed by some natural number in the code $z$, and for each such element we will start the algorithm which simulates the computation of $\varphi_{p}(0)$ for every program $p$, until a witness is produced for the given element. By hypothesis, we will eventually find a $y=\varphi_{p}(0)$ for some program $p$ such that $\psi\left(a, A_{y}\right)$ holds, and we copy the code $y$ to represent an element of the set $B$ we are building. After doing this for each element of the set coded by $z$, we have written a code for the set $B$, and we may halt. So $\Delta_{0}$-collection holds, and therefore the set of writable reals is a model of KP, and hence 
admissible.

Corollary 8.2 The supremum $\lambda$ of the writable ordinals is an admissible ordinal.

Proof: Every writable real is constructible because we could just run the computation in $L$. Also, if $\alpha$ is a writable ordinal, then we claim that $L_{\alpha}$ is coded by a writable real. This is true because, as in the Lost Melody Theorem 1.9, an infinite time Turing machine can simulate the construction of $L_{\alpha}$ given a code for $\alpha$; given a code for $L_{\beta}$, one obtains a code for $L_{\beta+1}$ by enumerating the definitions and systematically writing down the codes for the definable subsets of $L_{\beta}$; and at limit stages of the $L$ construction, given the sequence of codes for the earlier stages, one simply writes down the code for the union. Thus, if $\lambda$ is the supremum of the writable ordinals, $L_{\lambda}$ is a subclass of the collection of sets coded by writable reals (it is not clear whether these collections are distinct). Furthermore, the proof of the previous theorem can be modified to use $L_{\lambda}$ rather than the class of all sets coded by writable reals. Thus, rather than just searching for writable reals coding hereditarily countable sets, one searches for writable reals coding writable ordinals $\alpha$, and then uses these ordinals to construct $L_{\alpha}$ in the manner we have just explained, and then searches for the witnesses in these $L_{\alpha}$. Thus, $L_{\lambda}$ is admissible, and consequently $\lambda$ is admissible.

The next theorem will show that $\lambda$ is quite high up in the hierarchy of admissible ordinals. An ordinal $\beta$ is recursively inaccessible when it is an admissible limit of admissible ordinals. The ordinal $\beta$ is indescribable by a class of properties if there is a real $x$ coding $L_{\beta}$ such that for any property in the class which is true of $x$ there is an $\alpha<\beta$ and a real $y$ coding $L_{\alpha}$ having the very same property.

Indescribability Theorem 8.3 The supremum $\lambda$ of the writable ordinals is recursively inaccessible. Indeed, it is the $\lambda^{\text {th }}$ recursively inaccessible ordinal, and the $\lambda^{\text {th }}$ such fixed point, and so on. This is because $\lambda$ is indescribable by $\Pi_{1}^{1}$ properties. Indeed, $\lambda$ is indescribable by semi-decidable properties.

Proof: Let $\lambda$ be the supremum of the writable ordinals. We have already shown that $\lambda$ is admissible. If it is not a limit of admissible ordinals, then 
there is some largest admissible $\delta<\lambda$, which is consequently writable. Now, whether a real codes an admissible ordinal is infinite time decidable, since from the code for an ordinal $\alpha$ we have already explained how to get a code for the set $L_{\alpha}$, and it is decidable to check whether the set coded by a given real is admissible, since this consists in merely checking that the real codes a model of a certain recursively axiomatized theory. So, consider the supertask algorithm which first writes a real coding $\delta$ on a portion of the scratch tape, and then simultaneously simulates the computation of $\varphi_{p}(0)$ for every program $p$. For every real appearing during these computations, the algorithm checks to see if it codes an ordinal larger than $\delta$ which is admissible. If so, the algorithm gives that real as the output and halts. Since $\lambda$ is accidentally writable, this algorithm is bound to find, and write, the code for an admissible ordinal above $\delta$. By our assumption, this ordinal must be at least $\lambda$, contradicting the fact that $\lambda$ is the supremum of the writable ordinals. Thus, $\lambda$ must be an admissible limit of admissible ordinals.

By a similar argument we will now show that it cannot be the least such limit. Suppose $\lambda$ is the least limit of admissible ordinals above $\delta$. Now, it is a decidable question whether a real codes an ordinal which is an admissible limit of admissible ordinals; one simply tests first whether the real codes an admissible ordinal, and then tests whether there is any index for a smaller ordinal with no admissible ordinals in-between. So consider the supertask algorithm which first writes a code for $\delta$ on a portion of the scratch tape, and then searches for an accidentally writable real which codes an admissible limit of admissible ordinals, and tests if it is larger than $\delta$. When such a real is found, the algorithm gives it as the output and halts. By hypothesis, this algorithm will write a real at least as large as $\lambda$, a contradiction.

Suppose now that $\lambda$ is the $\delta^{\text {th }}$ admissible ordinal for some $\delta<\lambda$. Consider the algorithm which first writes $\delta$ on a portion of the scratch tape, and then searches for admissible ordinals which are coded by accidentally writable reals. Each time one is found which is larger than the previous ones, it is written on a portion of the scratch tape labeled with the index of the next element in the relation coding $\delta$. When every element of the field of that relation is taken care of, the algorithm writes the corresponding ordinal on the tape, and halts. By assumption, this real codes an ordinal at least as large as $\lambda$, a contradiction.

The indescribability argument is no different. Let $x$ be an eventually writable real which codes $L_{\lambda}$ in the manner of which we have been speaking. 
If $x$ has some semi-decidable property, then there must be a smaller ordinal $\alpha<\lambda$ with an accidentally writable real $y$ coding $L_{\alpha}$ with the very same property, since otherwise the algorithm which went searching for such a real would be able to write an ordinal larger than $\lambda$, which is impossible. In fact, there must be a writable $y$ with that property, since the algorithm will halt when one is found. Consequently, $\lambda$ is indescribable by semi-decidable properties.

Question 8.4 Is $\lambda$ the least ordinal which is indescribable by semi-decidable properties?

Next, we will prove the corresponding facts about the class of sets coded by eventually writable reals.

Theorem 8.5 The class of sets coded by eventually writable reals is admissible.

Proof: This proof is very similar to the corresponding proof for writable reals. But it also has the flavor of a finite injury priority argument in classical recursion theory. The class in question is closed under the rudimentary functions, and satisfies $\Delta_{0}$-comprehension just as before. The hard part is $\Delta_{0}$-collection. So suppose $z$ is eventually writable, and for every $a \in A_{z}$ there is a set $b$ coded by an eventually writable real such that $\psi(a, b)$, where $\psi$ is some fixed $\Delta_{0}$ formula. We have to collect witnesses into a set which is eventually writable. Consider the supertask algorithm which writes approximations to $z$ on a portion of the scratch tape. We will use these approximations to $z$ to write down a code for a set of witnesses. Eventually the correct approximation will be written, and we will eventually write down a code for a set of witnesses. So, for each approximation $w$ to $z$, we start writing down the code for a set of witnesses that will work for $w$, simultaneously computing better approximations to $z$. For each index $n$, representing a set $a$ in $A_{w}$, we search for an eventually writable witness $b$ for $a$. We do this by simulating for every program $p$ the computation of $\varphi_{p}(0)$ and testing the approximate outputs $b$ for these computations to see if $\psi(a, b)$ holds. If so, we copy the code for $b$ to be an element of $B$ on the true output tape. Periodically, however, the approximation $w$ may change because it has not 
yet stabilized, and when this occurs, we erase the witness $b$ from the output tape, and move to the approximation produced by another program, in such a way that we give every program a chance to produce the correct witness. Eventually, by hypothesis, we will hit on a witness which has stabilized. Furthermore, we do this simultaneously for each set $a \in A_{w}$, and every time the approximation $w$ changes, we start the process completely over with the new approximation. Eventually, $z$ will be written as its own approximation, and the witness computations will hit on the stabilizing witness which will be copied as elements of the set $B$ on the output tape. While the algorithm will continue searching for better approximations, it will never change the set $B$ after this point, and so we may collect witnesses into an eventually writable set $B$. So $\Delta_{0}$-collection holds.

Let us define that a set $A$ is eventually decidable when there is a program $p$ such that for any $x$ the computation $\varphi_{p}(x)$ never halts, but eventually has either a 1 or a 0 written on the output tape (never subsequently to change), respectively, depending on whether $x \in A$ or not. It is clear that every semi-decidable set is eventually decidable. Similarly, $A$ is eventually semidecidable when there is a program which for any $x$ eventually writes the Yes answers on the output tape, accordingly, depending on whether $x \in A$ or not.

Corollary 8.6 The supremum $\zeta$ of the eventually writable ordinals is an admissible ordinal, a recursively inaccessible ordinal, the $\zeta^{\text {th }}$ recursively inaccessible ordinal, the $\zeta^{\text {th }}$ such fixed point, and so on. It is indescribable by decidable properties and even by eventually semi-decidable properties.

Proof: Let $\zeta$ be the supremum of the eventually writable ordinals. By the argument of 8.3 it follows that $L_{\zeta}$ is a subclass of the sets coded by eventually writable reals (though again it is not clear whether these classes are distinct). In any case, the proof of the previous theorem is easily adapted as in 8.3 , by limiting the searches to $L_{\zeta}$ rather than any eventually writable set, to show that $L_{\zeta}$ is admissible. Consequently, $\zeta$ is an admissible ordinal.

Next, let us argue that $\zeta$ is accidentally writable, even though it is not eventually writable. Consider the supertask algorithm which simulates the computation of $\varphi_{p}(0)$ simultaneously for every program $p$. Our algorithm checks which of the output approximations code well orders, and writes a 
code for the sum of the ordinals coded by those that do. Eventually, the eventually writable ordinals are written as their own approximations, and so we have written an ordinal bigger than every eventually writable ordinal. Since the accidentally writable ordinals are closed under initial segment, it follows that $\zeta$ itself is accidentally writable.

Now we can show that $\zeta$ is recursively inaccessible, and indescribable, and so on, just as before. There is an accidentally writable real $x$ coding $L_{\zeta}$. Suppose that $x$ has some eventually semi-decidable property. Consider the algorithm which searches for reals with this very same property, and writing the real coding the corresponding ordinal. If $\zeta$ is the least ordinal such that $L_{\zeta}$ is coded by an accidentally writable real with that property, then this algorithm will eventually settle on a real coding an ordinal at least as large as $\zeta$, contradicting the fact that $\zeta$ is the supremum of all eventually writable ordinals. Thus, there must be a smaller $\alpha<\zeta$ with an accidentally writable real $y$ coding $L_{\alpha}$. The real $y$ will be eventually writable, since the algorithm will settle on $y$. So $\zeta$ is indescribable by eventually semi-decidable properties.

In our penultimate theorem, let us give necessary and sufficient conditions on the question of whether every clockable ordinal is writable. We have gone back and forth on this issue, and we simply do not know the answer. As usual, $\lambda$ is the supremum of the writable ordinals, and $\gamma$ is the supremum of the clockable ordinals.

Theorem 8.7 The following are equivalent:

1. $\lambda=\gamma$.

2. Every clockable ordinal is writable.

3. $\lambda$ is a limit of clockable ordinals.

4. The halting problem $h_{\lambda}$ is not decidable.

5. $\gamma$ is admissible.

Proof: $(1 \leftrightarrow 2)$ This is clear, since there are no gaps in the writable ordinals, and certainly $\lambda \leq \gamma$, since $\lambda$ is the order-type of the clockable ordinals. 
$(1 \leftrightarrow 3)$ The forward direction is clear, since $\gamma$ is a limit of clockable ordinals. For the converse direction, suppose that $\lambda$ is a limit of clockable ordinals, but $\lambda<\gamma$. Thus, $\lambda$ begins a gap in the clockable ordinals (recall that $\lambda$ itself is not clockable by 3.8). Let $\bar{\lambda}$ be the supremum of the ordinals which are writable in less than $\lambda$ many steps of computation. The lengths of these computations must in fact be unbounded in $\lambda$, since otherwise they would be bounded by some clockable ordinal $\beta<\lambda$, and we could run them all at once, with a $\beta$-clock, and write a real coding an ordinal larger than $\bar{\lambda}$ in fewer than $\lambda$ many steps, contradicting the definition of $\bar{\lambda}$. Also, by essentially the same argument, it must be that $\bar{\lambda}<\lambda$ since otherwise we could run all the computations up to some clockable $\beta$ beyond $\lambda$ and write a real coding an ordinal larger than $\lambda$, a contradiction. Now we can complete the argument by observing that the map which takes $\alpha<\bar{\lambda}$ to the length of the shortest computation which writes $\alpha$ is an unbounded map from $\bar{\lambda}$ to $\lambda$. Since it is $\Sigma_{1}$-definable in $L_{\lambda}$, where the computations exist, this contradicts the fact that $\lambda$ is admissible.

$(1 \leftrightarrow 4)$ The forward direction again is clear since $h$ is not decidable. For the converse, observe that if $\lambda$ is below some least clockable ordinal $\beta$, then $h_{\lambda}=h_{\beta}$, and $h_{\beta}$ is decidable by Theorem 4.5.

$(1 \leftrightarrow 5)$ The forward direction follows since $\lambda$ is admissible. For the converse, suppose that $\lambda<\gamma$. By the argument showing $(3 \rightarrow 1)$, it must be that the lengths of the computations which write the various ordinals below $\lambda$ are unbounded in $\gamma$. Consequently, in $L_{\gamma}$ we have a $\Sigma_{1}$-definable map from $\lambda$ unbounded in $\gamma$. So $\gamma$ cannot be admissible.

The argument in $(1 \leftrightarrow 3)$ shows that if $\lambda<\gamma$, then there are no ordinals in the interval $[\lambda, \gamma]$ which are both admissible and a limit of clockable ordinals.

We would like to conclude our paper by answering a question we teased the reader with way back in section three. Namely, is the ordinal $\omega_{1}^{C K}$ clockable? The answer, by the following theorem, is No.

Theorem 8.8 No admissible ordinal is clockable.

Proof: Suppose that $\alpha$ is a clockable limit ordinal. We will show that $\alpha$ is not admissible by showing that there is a function $f: \omega \rightarrow \alpha$, unbounded in $\alpha$, which is $\Sigma_{1}$-definable in $L_{\alpha}$. It follows that $L_{\alpha}$ cannot model $\Sigma_{1}$-collection, and therefore cannot be admissible. It suffices to consider the case when $\alpha$ 
is a limit of limit ordinals. Let $p$ be a program which on input 0 halts in exactly $\alpha$ steps. And let us pay attention to exactly the manner in which the program halts at stage $\alpha$. At stage $\alpha$ the head is at the extreme left of the tapes and in the limit state. Furthermore, the values appearing in the three cells under the head have caused the machine to halt. Thus, this must be the first time at a limit ordinal that those cells have that particular pattern, or the machine would have halted earlier. The 0 s on the tape must have been 0 from some point on, and perhaps one of the 1s was also like that. But there must have been a 1 on the cell under the head which had alternated infinitely often before $\alpha$, since otherwise the snapshot at $\alpha$ would have been obtained at one of the limit stages before $\alpha$ (since by assumption such stages are unbounded in $\alpha$ ), and the program would have halted earlier. Furthermore, stage $\alpha$ must be the first stage by which that cell had alternated from 1 to 0 and back infinitely many times, since otherwise again the program would have halted earlier. Let $h(n)$ be the stage at which the cell alternated the $n^{\text {th }}$ time. We have argued that these stages are unbounded in $\alpha$. Furthermore, the function $h$ is $\Sigma_{1}$-definable in $L_{\alpha}$, since the initial segments of the computation of $\varphi_{p}(0)$ all live in $L_{\alpha}$, and are defined by a $\Sigma_{1}$ definition there. Thus, over $L_{\alpha}$ there is a $\Sigma_{1}$ definable map from $\omega$ unbounded in $\alpha$, and so $\alpha$ is not admissible.

The authors can be reached at the following addresses:

Joel David Hamkins, Mathematics, City University of New York, College of Staten Island, Staten Island, NY 10314; hamkins@postbox.csi.cuny.edu

Andy Lewis, Mathematics, Virginia Commonwealth University, Box \#842014, Richmond, Va. 23284-2019; amlewis@saturn.vcu.edu

\section{References}

[Bar] John Barwise, Admissible Set Theory, Springer Verlag Publishing Company, New York, (1990).

[Dub] Derrick Albert Dubose, The Equivalency of Determinacy and Iterated Sharps, Journal of Symbolic Logic (June, 1980) Volume 55, Number 
$2,502-510$.

[Ear \& Nor] John Earman and John D. Norton, Forever is a day: supertasks in Pitowski and Malament-Hogarth spacetimes, Philos. Sci., 60 (1993) no. $1,22-42$

[Ear] John Earman, Bangs, crunches, whimpers and shrieks: singularities and acausalities in relativistic spacetimes. The Clarendon Press, Oxford University Press, New York, (1995).

[Fef \& Spc] Solomon Feferman and C. Spector, Incompleteness along paths in progressions of theories, Journal of Symbolic Logic 27 (1962) p. 383390 .

[Hog92] Hogarth, Does general relativity allow an observer to view an eternity in a finite time?, Foundations of Physics Letters, 5, p 173-181 (1992).

[Hog94] Hogarth, Non-Turing computers and non-Turing computability, in PSA 1994, Vol 1 East Lansing: Philosohpy of Science Association, (D. Hull, M. Forbes, and R.B Burian, eds.) 126-138.

[Mos] Yiannis Nicholas Moschovakis, Descriptive Set Theory, North-Holland Publishing Company, New York, (1980).

[Pit] Pitowsky, The physical Church Thesis and Physical computational complexity, Iyyun, 39, pp. 81-99 (1990).

[Sacks] Gerald E. Sacks, Higher Recursion Theory, Springer Verlag Publishing Company, New York, (1990).

[Soare] Robert I. Soare, Recursively Enumerable Sets and Degrees, Springer Verlag Publishing Company, New York, (1987).

[Thom] Thomson, Tasks and Super-tasks, Analysis, XV, p.1-13 (1954-55). 